HUTP-05/A0030

UTAP-530

RESCEU-10/05

\title{
Dynamics of Gravity in a Higgs Phase
}

\author{
Nima Arkani-Hamed ${ }^{\mathrm{a}}$, Hsin-Chia Cheng ${ }^{\mathrm{a}, \mathrm{b}}$, Markus A. Luty ${ }^{\mathrm{a}, \mathrm{c}, \mathrm{d}}$, \\ Shinji Mukohyama ${ }^{\mathrm{a}, \mathrm{e}}$, Toby Wiseman ${ }^{\mathrm{a}}$ \\ a Jefferson Laboratory of Physics, Harvard University \\ Cambridge, Massachusetts 02138 \\ ${ }^{\mathrm{b}}$ Department of Physics, University of California \\ Davis, California 95616 \\ c Physics Department, Boston University \\ Boston, Massachusetts 02215 \\ ${ }^{d}$ Physics Department, University of Maryland \\ College Park, Maryland 20742* \\ ${ }^{\mathrm{e}}$ Department of Physics and Research Center for the Early Universe \\ The University of Tokyo, Tokyo 113-0033, Japan
}

\begin{abstract}
We investigate the universal low-energy dynamics of the simplest Higgs phase for gravity, 'ghost condensation.' We show that the nonlinear dynamics of the 'ghostone' field dominate for all interesting gravitational sources. Away from caustic singularities, the dynamics is equivalent to the irrotational flow of a perfect fluid with equation of state $p \propto \rho^{2}$, where the fluid particles can have negative mass. We argue that this theory is free from catastrophic instabilities due to growing modes, even though the null energy condition is violated. Numerical simulations show that solutions generally have singularities in which negative energy regions shrink to zero size. We exhibit partial UV completions of the theory in which these singularities are smoothly resolved, so this does not signal any inconsistency in the effective theory. We also consider the bounds on the symmetry breaking scale $M$ in this theory. We argue that the nonlinear dynamics cuts off the Jeans instability of the linear theory, and allows $M \lesssim 100 \mathrm{GeV}$.
\end{abstract}

\footnotetext{
${ }^{*}$ Permanent address
} 


\section{Introduction}

Is general relativity the correct description of gravity at long distances and times? Certainly, there are good reasons for thinking that this is the case. Experimentally, gravity has been probed at distance scales ranging from $10^{-1} \mathrm{~mm}$ (in short-range force experiments) to at least $10^{14} \mathrm{~cm}$ (the size of the solar system). Theoretically, general relativity is the unique Lorentz-invariant theory of massless spin 2, and its conceptual elegance is beyond question. However, the situation is far less clear on cosmological distance and time scales. Structure formation, galaxy rotation curves and gravitational lensing, and the accelerating expansion of the universe cannot be explained by general relativity coupled to known matter. These anomalous effects are conventionally attributed to 'dark matter' and 'dark energy.' However, given the fact that the observed effects are purely gravitational, it makes sense to ask whether they may have a common origin in a modification of gravity in the infrared. These considerations have led to a revival of interest in consistent infrared modifications of gravity $[1,2,3,4,5,6,7]$.

In the present paper, we further investigate the model of Ref. [5], 'ghost condensation.' This can be viewed as the universal low-energy dynamics associated with the simplest Higgs phase for gravity, arising when Lorentz symmetry is broken spontaneously. Breaking of Lorentz symmetry is of course ubiquitous. For example, timedependent fields in cosmology define a preferred frame. However, these solutions are not the ground state of the theory: they carry energy density and dilute away as the universe expands. Any 'modification of gravity' induced by such solutions becomes relevant only at scales of order the horizon. We are instead interested in the situation where Lorentz symmetry is broken in flat spacetime, allowing nontrivial modification of gravity inside the horizon. This means that the symmetry breaking sector has peculiar properties; in particular, the stress-energy tensor must vanish in the ground state:

$$
\left\langle T_{\mu \nu}\right\rangle=0 .
$$

Spontaneous breaking of Lorentz symmetry gives rise to a gapless scalar excitation analogous to the Nambu-Goldstone bosons that arise from spontaneous breaking of internal symmetries. 'Ghost condensation' gives rise to a single such mode, and is in this sense the minimal model of spontaneous breaking of Lorentz symmetry. We refer to the scalar mode as a 'ghostone boson.' In analogy with the Higgs phase for gauge theory, the ghostone mode mixes with the graviton, modifying gravity in a nontrivial manner. 
Ref. [5] studied this theory and analyzed the modification of gravity in the weakfield limit. The dynamics is governed by a consistent effective theory defined by the scale $M$ where the symmetry is broken. It was shown that the ghostone mode gives a possible new origin for dark energy and dark matter. Ref. [8] showed that the ghostone mode may also be the inflaton, leading to interesting testable consequences.

In the present paper, we further investigate the dynamics of this theory. We show that nonlinearities dominate the dynamics of the ghostone sector for all gravitational sources of interest. In particular, the time scale for the onset of nonlinear dynamics for a fixed gravitational source is precisely the infall (or orbit) time associated with the source. Away from singularities, the nonlinear solutions are equivalent to the gradient flow of a fluid with equation of state

$$
p=\frac{\rho^{2}}{2 M^{4}} .
$$

The gradient fluid flow picture breaks down at caustic singularities where geodesics of fluid particles cross. At these points higher-derivative terms become important. Numerical simulations show that these remove the caustic singularity in most cases, but in some cases singularities still form. These correspond to regions of negative energy that shrink in size until their dynamics becomes sensitive to the underlying microscopic theory. We argue that these can be stabilized in a partial UV completion of the model into 'gauged ghost condensation.'

We also address stability issues in the nonlinear theory. Although the energy is not positive-definite in the nonlinear theory, there are no growing modes corresponding to infinite positive energy radiated to infinity. We argue that the worst instability is that of negative energy regions shrinking in size, as found in the simulations.

Based on these results, we address the question of the bounds on the ghostone sector. We find that the most sensitive bounds come from the fact that the Jeans instability found in Ref. [5] produces regions of positive and negative energy and that those regions induce scintillation of light rays coming from far distances by gravitational lensing. Demanding that the total angular deviation by the scintillation, or the random walk, not exceed the observed angular resolution gives the bound $M \lesssim 100 \mathrm{GeV}$. Other possible observable effects such as modification of gravity near astrophysical sources and energy loss of moving sources are argued to be negligibly small. Black holes in the ghost condensate are studied in Ref. [9], where it is found that they also do not give strong constraints on the model (see also Ref. [10]).

This paper is organized as follows. In section 2, we review the Higgs phase for gravity, emphasizing the universal nature of the low-energy dynamics. We also review the origin of the relevant length and time scales in the linearized theory. In section 
3, we discuss the nonlinear dynamics of the ghost condensate. We discuss the time scales and present the fluid picture. In section 4 , we show the numerical simulations of the nonlinear evolutions and discuss the resolution of caustic singularities. In section 5 , we discuss the bounds of this theory. This includes mass and energy accretion in slow-moving objects, gravitational lensing and energy loss from moving objects. We do not claim to have a complete understanding of the dynamics, so this section is intended to be preliminary and provocative. In section 6 , we briefly discuss the possibility that ghost condensate may constitute the dark matter. We show that the initial growth of the density perturbations in the linear regime is identical to that of the standard cold dark matter. Whether it can form the correct structure depends on the details of the nonlinear evolution which is left for future investigations. Our conclusions are presented in section 7 .

\section{Review of the Linear Theory}

\subsection{Effective Theory}

What is a Higgs phase for gravity? It is easiest to answer this question in linearized general relativity, where we expand the metric about flat space

$$
g_{\mu \nu}=\eta_{\mu \nu}+h_{\mu \nu}
$$

and keep only terms quadratic in $h_{\mu \nu}$. In this theory, the fields $h_{\mu \nu}$ are closely analogous to gauge fields with gauge transformation law

$$
\delta h_{\mu \nu}=-\left(\partial_{\mu} \xi_{\nu}+\partial_{\nu} \xi_{\mu}\right)
$$

where $\xi^{\mu}$ are the generators of infinitesmal diffeomorphisms

$$
x^{\mu} \mapsto x^{\mu}+\xi^{\mu}
$$

We want to consider the case where the time diffeomorphisms generated by $\xi^{0}$ are spontaneously broken. This means that time diffeomorphisms are realized nonlinearly

in the effective theory containing only the ghostone field. The minimal model contains a single real ghostone field $\pi$ that shifts under time diffeomorphisms:

$$
\delta \pi=-\xi_{0}
$$

Note that $\pi$ naturally has units of time. We now write the most general effective Lagrangian invariant under these symmetries. This contains the Einstein Lagrangian, 
and additional terms constructed from the invariants

$$
\begin{aligned}
\Sigma & =\dot{\pi}-\frac{1}{2} h_{00}, \\
K_{i j} & =\frac{1}{2}\left(\dot{h}_{i j}-\partial_{i} h_{0 j}-\partial_{j} h_{0 i}+2 \partial_{i} \partial_{j} \pi\right) .
\end{aligned}
$$

The leading terms are

$$
\mathcal{L}_{\text {eff }}=\mathcal{L}_{\mathrm{E}}+M^{4}\left\{\frac{1}{2}\left(\dot{\pi}-\frac{1}{2} h_{00}\right)^{2}-\frac{\alpha_{1}}{2 M^{2}} K_{i j}^{2}-\frac{\alpha_{2}}{2 M^{2}} K^{2}+\mathcal{O}\left(\pi^{3}\right)\right\} .
$$

(Note that we have built in the fact that flat space is a solution by not writing any linear terms in the Lagrangian.)

In the limit where we turn off gravity, we see that the ghostone mode has dispersion relation

$$
\omega^{2}=\frac{\alpha \vec{k}^{4}}{M^{2}}
$$

where $\alpha=\alpha_{1}+\alpha_{2}$. If the dimensionless couplings in the effective Lagrangian are order 1 , the mass scale $M$ is the scale of new physics in this theory.

Eq. (2.7) is the starting point for analyzing the linear dynamics of the theory, including the coupling to gravity. However, there are several points that may not be completely clear in this formulation. First, it may not be completely clear in what sense the breaking of Lorentz symmetry is spontaneous, since the time direction appears to be explicitly singled out. Second, it is not clear whether the absence of a linear term in $\Sigma$ (allowed by all symmetries) requires an additional fine-tuning, like the cosmological constant which is a linear term proportional to $h_{\mu}^{\mu}$. Third, we would like to have an efficient way to extend this to the full nonlinear theory. All of these issues can be elegantly understood in the covariant formulation, which we turn to next.

\subsection{Covariant Formulation}

Consider an effective theory with a real scalar $\phi$ that is invariant under a global shift symmetry

$$
\delta \phi=\lambda
$$

where $\lambda$ is a spacetime independent constant. For example, $\phi$ could be a NambuGoldstone boson from the breaking of a global symmetry, or a 0-form gauge field in string theory. 
A conventional effective Lagrangian for this theory is

$$
\mathcal{L}=+\frac{1}{2} \partial^{\mu} \phi \partial_{\mu} \phi
$$

with equation of motion $\square \phi=0$. This has solutions with $\phi=n_{\mu} x^{\mu}$ for any constant 4 -vector $n_{\mu}$. If $n_{\mu}$ is timelike, we can choose the time direction so that the solution is

$$
\phi=c t
$$

This is a solution for any constant $c$. At first sight it may appear that these are obviously not candidate ground states of the theory, but the situation is actually more subtle. Suppose we expand in fluctuations about this solution

$$
\phi=c t+\pi
$$

Note that under time diffeomorphisms, $\pi$ transforms as

$$
\delta \pi=-\xi_{0} / c
$$

just like the ghostone mode considered above. Expanding the Lagrangian to quadratic order in $\pi$, one finds that the fluctuations for $\pi$ have good time and space kinetic terms. This means that the solution is stable under local fluctuations for any value of $c$ ! The reason for this is that the theory has a conserved current associated with the shift symmetry

$$
J_{\mu}=\partial_{\mu} \phi
$$

Solutions with $c \neq 0$ have a constant nonzero charge density. Local excitations cannot change the total charge, so configurations with lower energy cannot be reached. However, when we turn on gravity, solutions with $c \neq 0$ will cause the universe to expand, and the charge will dilute away. Lorentz invariance is therefore not broken spontaneously in this theory.

Consider instead an effective Lagrangian of the form

$$
\mathcal{L}_{\text {eff }}=M^{4} P(X), \quad X=\partial^{\mu} \phi \partial_{\mu} \phi
$$

Note that $\phi$ has dimensions of length (or time), so that $X$ is dimensionless. This omits only terms with more than one derivative acting on $\phi$, such as $(\square \phi)^{2}$. We will include their effects below. This also has solutions of the form $\phi=c t$ for any $c$. Expanding in fluctuations Eq. (2.12), we obtain

$$
\mathcal{L}_{\text {eff }}=M^{4}\left\{\left[2 c^{2} P^{\prime \prime}\left(c^{2}\right)+P^{\prime}\left(c^{2}\right)\right] \dot{\pi}^{2}-P^{\prime}\left(c^{2}\right)(\vec{\nabla} \pi)^{2}+\mathcal{O}\left(\pi^{3}\right)\right\}
$$


We see that small perturbations are stable provided

$$
2 c^{2} P^{\prime \prime}\left(c^{2}\right)+P^{\prime}\left(c^{2}\right)>0, \quad P^{\prime}\left(c^{2}\right)>0 .
$$

(Note that a conventional kinetic term $P(X)=+\frac{1}{2} X$ satisfies these conditions.)

To see whether any of these solutions may be regarded as candidate ground states, we compute the stress-energy tensor:

$$
T_{\mu \nu}=M^{4}\left[-P(X) g_{\mu \nu}+2 P^{\prime}(X) \partial_{\mu} \phi \partial_{\nu} \phi\right]
$$

In the solution $\phi=c t$, the first term is proportional to $g_{\mu \nu}$ and can be cancelled by tuning the cosmological constant. The second term gives rise to

$$
T_{00}=2 c^{2} P^{\prime}\left(c^{2}\right), \quad T_{i j}=0
$$

This gives rise to an expanding universe unless

$$
P^{\prime}\left(c^{2}\right)=0
$$

Note that the conserved shift current is given by

$$
J_{\mu}=2 P^{\prime}(X) \partial_{\mu} \phi
$$

so the charge density vanishes in this configuration. Therefore, if $P(X)$ has the form shown in Fig. 1, then the configuration with $P^{\prime}(X)=0$ is a candidate ground state. This is the 'ghost condensate.' From now on, we rescale the field $\phi$ so that the ground state is at $X=1$, and write

$$
\Sigma=\frac{1}{2}(X-1)
$$

Stability in the linearized theory requires higher-derivative terms to give a nonzero spatial kinetic term, for example

$$
\Delta \mathcal{L}_{\text {eff }}=-\frac{\alpha M^{2}}{2}(\square \phi)^{2}=-\frac{\alpha M^{2}}{2}\left(\vec{\nabla}^{2} \pi\right)^{2}+\cdots .
$$

This leads to the $\omega^{2} \sim \vec{k}^{4}$ dispersion relation for the ghostone mode already discussed above. 


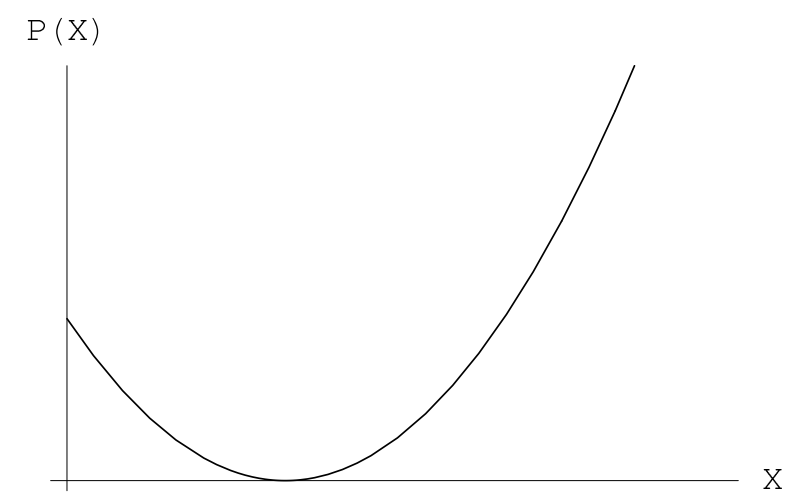

Fig. 1. The kinetic fuction $P(X)$ for ghost condensation.

\subsection{Higgs Phase for Gravity}

The ghostone mode mixes with gravity, giving rise to a nontrivial modification of gravity in the infrared. A detailed analysis is given in Ref. [5], but much of the physics can be understood from very general considerations, which we review here.

Recall first the ordinary Higgs mechanism for gauge theory. In this case, a nonzero charge gives rise to a tadpole for the Goldstone mode. Excitations of the Goldstone mode themselves carry charge, and because opposite charges attract, the Goldstone charge tends to screen the original charge. If the charge configuration is timedependent, the Goldstone field responds at the speed of light. This is illustrated schematically in Fig. 2.

Similarly, in the Higgs mechanism for gravity, a nonzero mass (the charge that sources gravity) gives rise to a tadpole for the ghostone mode. Excitations of the ghostone mode themselves carry energy, but because positive masses attract in gravity, the ghostone field anti-screens the source gravitational field. This gives rise to an instability of the vacuum analogous to the Jeans instability of pressureless matter coupled to gravity. The ghostone Jeans instability can be seen in the dispersion relation for the scalar mode that follows from the mixing with gravity:

$$
\omega^{2}=\frac{\alpha \vec{k}^{4}}{M^{2}}-\frac{\alpha M^{2}}{2 M_{\mathrm{Pl}}^{2}} \vec{k}^{2} .
$$

This is illustrated schematically in Fig. 3. The length and time scale associated with the Jeans instability are

$$
L_{\mathrm{J}} \sim \frac{M_{\mathrm{Pl}}}{M^{2}}, \quad T_{\mathrm{J}} \sim \frac{M_{\mathrm{Pl}}^{2}}{M^{3}} \gg L_{\mathrm{J}} .
$$




\section{Gauge Higgs Phase}

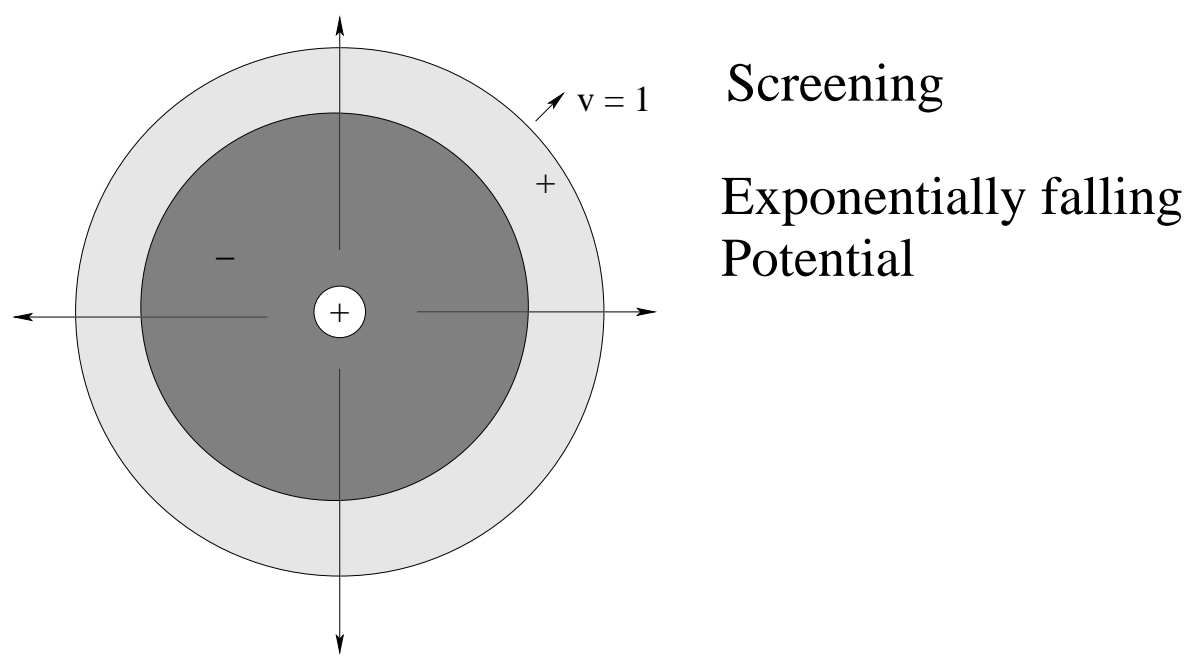

Fig. 2. A schematic illustration of the screening effect for gauge theories in the Higgs phase.

For $M \lesssim 10 \mathrm{MeV}, T_{\mathrm{J}}$ is longer than the lifetime of the universe and there is clearly no constraint from the Jeans instability.

\subsection{Negative Energy}

Even for time shorter than the Jeans time scale $T_{\mathrm{J}}$, stability is an issue because the ghostone energy can be negative. Recall that the ground state $X=1$ is the boundary of the stability region. Expanding to higher orders in $\pi$, we find interaction terms such as

$$
\mathcal{L}_{\text {eff }}=M^{4}\left[\frac{1}{2} \dot{\pi}^{2}-\frac{1}{2} \dot{\pi}(\vec{\nabla} \pi)^{2}+\cdots-\frac{\alpha}{2 M^{2}}\left(\vec{\nabla}^{2} \pi\right)^{2}\right] .
$$

In regions where $\dot{\pi}<0$ the cubic term gives rise to negative gradient energy, and therefore an instability. Classically, the $\left(\vec{\nabla}^{2} \pi\right)^{2}$ term restores stability at short wavelengths, and there is a growing mode only for long wavelengths:

$$
\lambda \gtrsim \frac{M^{-1}}{\langle\dot{\pi}\rangle^{1 / 2}} .
$$

Quantum-mechanically, there are fluctuations at all length scales. Nonetheless, Ref. [5] showed that there is no quantum instability in the effective theory using a scaling argument. If we scale energy by $E \rightarrow s E$, the quadratic kinetic terms are left invariant 


\section{Gravity Higgs Phase}

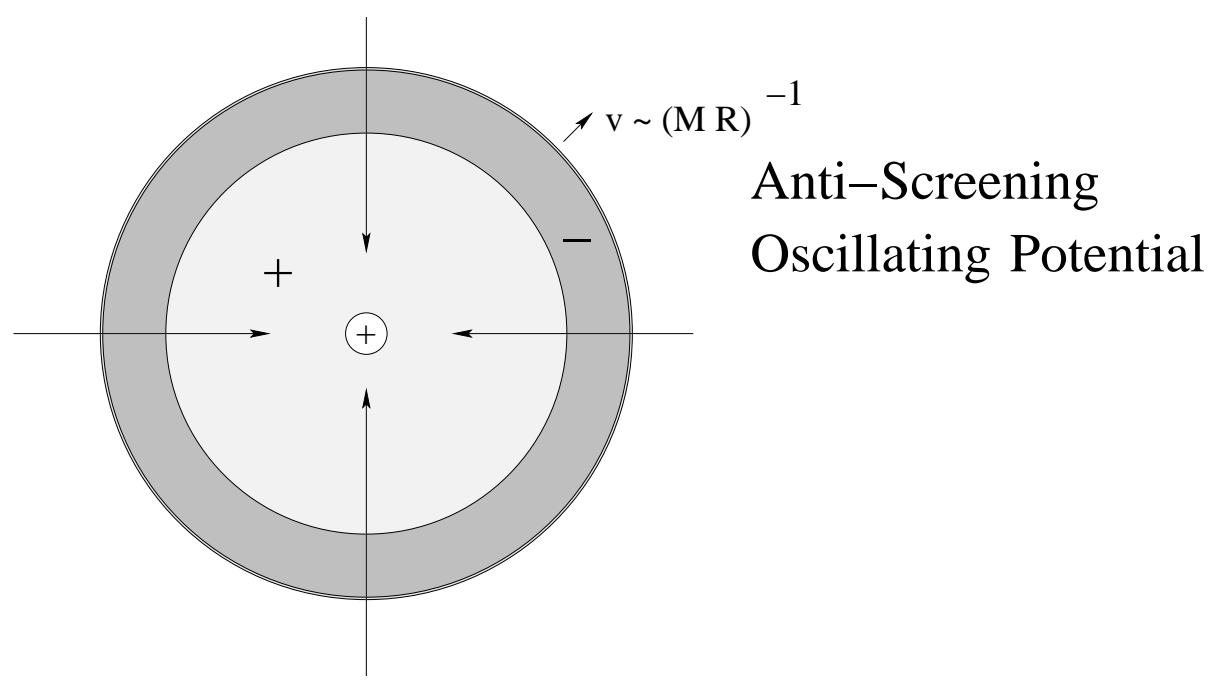

Fig. 3. A schematic illustration of the anti-screening effect for gravity in the Higgs phase

if we scale

$$
\begin{aligned}
t & \rightarrow s^{-1} t, \\
\vec{x} & \rightarrow s^{-1 / 2} \vec{x}, \\
\pi & \rightarrow s^{1 / 4} \pi .
\end{aligned}
$$

With this scaling the cubic operator $\dot{\pi}(\vec{\nabla} \pi)^{2}$ in Eq. (2.26) scales as $s^{1 / 4}$ and is therefore (barely!) irrelevant. All other operators are even more irrelevant, showing that there is a regime of low energies where the expansion is under control.

\subsection{Preview of Nonlinear Effects}

The arguments above show that the effects of the nonlinear terms are under control at low energies and small field amplitudes. However, in the presence of large classical gravitational sources the nonlinear terms can become important. In fact, the time scale for the ghostone field near a classical source is just the gravitational infall time of the source. This can be understood from the form of the stress-energy tensor. In the approximation where the Lagrangian is $\mathcal{L}=P(X)$, the stress-energy tensor has the form

$$
T_{\mu \nu} \propto-P(X) g_{\mu \nu}+2 P^{\prime}(X) u_{\mu} u_{\nu}
$$


where

$$
u_{\mu}=\partial_{\mu} \phi
$$

This has the form of a stress-energy tensor for a perfect fluid with 4-velocity $u_{\mu} \cdot{ }^{1}$ The fact that $u_{\mu}$ is a gradient means that the flow is irrotational: $\partial_{[\mu} u_{\nu]}=0$ implies $\vec{\nabla} \times \vec{u}=0$. The equations of motion for the ghostone field follow from the conservation of the stress-energy tensor $\nabla^{\mu} T_{\mu \nu}=0$, which are intepreted as the conservation of energy and momentum in the fluid. In the presence of a classical gravitational source, a fluid clearly responds on a time scale given by the infall time, and therefore so does the ghostone mode. As it will be shown in the next section, this is exactly the time scale where the nonlinear term becomes important.

For small fluctuations about the minimum $X=1, P(X)$ can be approximated by

$$
P(X) \approx \frac{1}{8}(X-1)^{2}=\frac{1}{2} \Sigma^{2}
$$

where an overall contribution to the cosmological constant has been removed. We can read off the equation of state of the fluid from the energy-momentum tensor. It is

$$
p=\frac{\rho^{2}}{2 M^{4}}
$$

where

$$
\rho=M^{4} \Sigma
$$

For $\rho \ll M^{4}$, small ghostone fluctuations therefore behave like an almost pressureless fluid, which makes the ghostone excitations a candidate for the dark matter in our universe. We will have more to say on this later.

As we noted above, Hubble expansion drives $\Sigma \rightarrow 0$, and therefore $\rho \rightarrow 0$. Even for $\rho=0$ the fluid has nontrivial dynamics in the presence of gravitational sources: the fluid particles follow geodesics. As a result, the local preferred frame (where $u_{\mu}=\partial_{\mu} \phi$ is 'at rest') is a freely-falling frame. This frame is also the preferred frame from the spontaneous breaking of Lorentz invariance, so we conclude that the 'aether' is dragged by the local gravitational field.

Since geodesics generically cross (especially in the presence of gravitational sources), the field configuration develops caustic singularities where the field gradients diverge.

\footnotetext{
${ }^{1}$ The defining property of a perfect fluid is that at each point there is a frame in which the stress-energy tensor has the form $T_{\mu \nu}=\operatorname{diag}(-\rho, p, p, p)$.
} 
Such phenomena were also observed in some other scalar field theories [11]. Near these singularities, the higher-derivative $\alpha\left(\vec{\nabla}^{2} \pi\right)^{2}$ term can no longer be neglected. This term contributes positive gradient energy, and we find in numerical simulations that it generically smoothly resolves the caustic singularity, giving rise to a 'bounce' with outgoing $\pi$ waves and positive and negative regions of $\Sigma$ near the would-be caustic.

The fact that $\Sigma$ (and hence $\rho$ ) can be negative brings up again the question of the stability of the theory. As discussed above, regions with $\Sigma<0$ modes with sufficiently long wavelengths are unstable. In numerical simulations, we find that negative energy regions tend to shrink while the amplitude of $\Sigma$ grows inside the region. This can be understood from the fluid picture, since this is valid in the limit where we neglect the $\left(\vec{\nabla}^{2} \pi\right)^{2}$ term, which is a good approximation away from caustic singularities. In this picture the $\Sigma<0$ region consists of fluid particles with negative mass. It is therefore clear that energy (mass) cannot flow across the boundary of the $\Sigma<0$ region, since the boundary consists of particles with vanishing mass. The boundary can move however, and in the $\Sigma<0$ region the positive pressure favors large gradients and causes the $\Sigma<0$ region to shrink. Numerical simulations show that some $\Sigma<0$ regions continue to shrink until they exit the regime of validity of the effective theory. These singularties need to be resolved in a more fundamental theory. Similar conclusion was also obtained in Ref. [12] which studied the twodimensional case. However, we show that the total energy inside the $\Sigma<0$ regions formed in astrophysical situations is very small, and does not lead to any observable consequences provided the singularities are regulated in a smooth way. We will discuss a partial UV completion to do this, and present numerical evidence that it works.

The nonlinear dynamics affects the bounds on $M$ derived in the linear theory. The Jeans instability in the linear theory gives a bound $M \lesssim 10 \mathrm{MeV}$ if we require that there is no exponential growth of the oscillatory potential within the age of the universe. However, the nonlinear dynamics is expected to cut off the instability, and may weaken this bound. We argue below that the strongest bound comes from gravitational lensing due to regions of positive and negative energy produced by the Jeans instability. Demanding that the random walk of light rays due to the lensing does not smear out the observed CMB anisotropies gives the bound $M \lesssim 100 \mathrm{GeV}$.

We also consider other possible bounds on the ghost condensate from the gravitational sector. The modifications of the gravitational potential are small due to velocity effects $[13,14]$. We also consider energy loss in the nonlinear theory, as well as energy stored in would-be caustic singularities. We find that these effects are negligible, and we believe that the theory is safe for $M \lesssim 100 \mathrm{GeV}$. 


\section{Nonlinear Dynamics}

We now turn to the nonlinear dynamics of the theory. The nonlinear dynamics is very rich and complex, and we emphasize that we do not claim a complete understanding in this work. It is therefore important to keep in mind that there is a simple limit of this theory, independent of the details of the nonlinear dynamics [5]. The Ghostone sector naturally couples to matter only through gravity. (Gravitationally induced direct couplings to standard model fields are easily seen to be negligibly small.) The maximum value of the gravitational energy in the Ghostone energy is of order $M^{4}$, which does not affect even cosmology if $M \lesssim\left(M_{\mathrm{Pl}} H_{0}\right)^{1 / 2} \sim 10^{-3} \mathrm{eV}$. Such low values of $M$ are still very interesting for cosmology, since the ghost may be a source of dark energy and dark matter [5] and may drive inflation [8].

Another general point to keep in mind in the following is that the modifications of gravity vanish in regions where $\Sigma=0(X=1)$ and we neglect the $\left(\vec{\nabla}^{2} \pi\right)^{2}$ term. This is because in this limit, the Lagrangian in 'unitary gauge' $\phi=t(\phi=0)$ is

$$
\mathcal{L}=\sqrt{-g} P\left(g^{00}\right) .
$$

This has the form of a gauge-fixing term for the gauge $X=g^{00}=1$, so if $X=1$ initially, the gravitational dynamics is unchanged. This means that any modification of gravity will be suppressed by the small amplitude $\Sigma$ or by the $\alpha\left(\vec{\nabla}^{2} \pi\right)^{2}$ term which is small at long wavelengths. We will see that this suppresses many possible effects compared to naïve expectations.

In the remainder of the paper, we consider $M \gg 10^{-3} \mathrm{eV}$ to see what modifications of gravity may be observable today, and determine the experimental limit on the scale M.

\subsection{Effective Lagrangian}

We are interested in the gravitational dynamics of large, slow-moving sources, where relativistic and retardation effects are negligible. We further restrict ourselves to the domain of weak gravity, so we are not considering black holes. (Black holes in the presence of the ghost condensate are discussed in Ref. [9].) It is convenient to incorporate these approximations from the beginning in an effective Lagrangian. The fact that gravity is weak means that we use the linearized approximation for gravity. The fact that the system is nonrelativistic means that $\partial_{t} \ll \vec{\nabla}$. For the motions of objects, we can neglect gravitational radiation, so we can parameterize the metric by the scalar potential $\Phi$ as

$$
d s^{2}=(1+2 \Phi) d t^{2}-(1-2 \Phi) d \vec{x}^{2}
$$


where

$$
\vec{\nabla}^{2} \Phi=\frac{T_{00}}{2 M_{\mathrm{Pl}}^{2}}
$$

We also assume that ghostone amplitudes are small in units of $M$. Since only derivatives of $\pi$ are meaningful, this means that

$$
|\vec{\nabla} \pi| \ll 1
$$

With these approximations, the deviation of $X$ from its minimum is given by

$$
\Sigma=\frac{1}{2}(X-1)=\dot{\pi}-\frac{1}{2}(\vec{\nabla} \pi)^{2}-\Phi
$$

Note that there is nothing in the assumptions above that determines the relative size of the terms on the right-hand side. The effective Lagrangian is then

$$
\mathcal{L}_{\text {eff }}=M^{4}\left\{\frac{1}{2} \Sigma^{2}-\frac{\alpha}{2 M^{2}}\left(\vec{\nabla}^{2} \pi\right)^{2}\right\}
$$

All the terms omitted are smaller than the ones we have kept by the assumptions above. Note that all the nonlinear effects in this approximation are due to the $(\vec{\nabla} \pi)^{2}$ term in $\Sigma$.

\subsection{Nonlinear Time Scale}

We now estimate the time scale for the nonlinear gradient terms in Eq. (3.5) to dominate the dynamics. We assume that the gravitational potential $\Phi$ is determined by external sources, and varies on a length scale $L$ in the region of interest. (For example, at a distance $r$ outside a gravitational source, $L \sim r$.) If initially $\pi=0$, the gravitational potential gives a tadpole that forces $\pi$ to be nonzero. We want to know the time scale $T_{\mathrm{NL}}$ when the nonlinear term in $\Sigma$ becomes as important as the linear $\dot{\pi}$ term. This is determined by

$$
\frac{\pi}{T_{\mathrm{NL}}} \sim \frac{\pi^{2}}{L^{2}} \sim \Phi
$$

where $\pi$ is a typical ghostone amplitude. This gives

$$
T_{\mathrm{NL}} \sim \frac{L}{\sqrt{\Phi}}
$$

We recognize this as the gravitational response time associated with the potential $\Phi$. For example, for a point source $\Phi \sim R_{\mathrm{S}} / r$ where $R_{\mathrm{S}}$ is the Schwarzschild radius, and 
we have $T_{\mathrm{NL}}^{2} \sim r^{3 / 2} / R_{\mathrm{S}}$, which is the Kepler relation. This is a very direct way of seeing that the nonlinear effects become important on the gravitational time scale.

Solving Eq. (3.7) for $\pi$, we obtain

$$
|\vec{\nabla} \pi| \sim \frac{\pi}{L} \sim \sqrt{\Phi} \ll 1 .
$$

That is, ghostone amplitudes are small for weak gravity, in agreement with our assumptions.

On the other hand, in the linear approximation the $\alpha\left(\vec{\nabla}^{2} \pi\right)^{2}$ becomes comparable to $\dot{\pi}^{2}$ at $T_{L i n} \sim M L^{2}$. As a result, the nonlinear evolutions completely dominates for $T_{N L} \lesssim T_{L i n} \sim M L^{2}$, or

$$
\Phi L^{2} \gtrsim \frac{1}{M^{2}} .
$$

For example, outside a gravitational source of mass $M_{\text {src }}$, this condition is

$$
r \gtrsim \frac{1}{M_{\mathrm{src}}}\left(\frac{M_{\mathrm{Pl}}}{M}\right)^{2},
$$

and the earth's surface gravity is in the nonlinear regime for $M \gtrsim 10^{-8} \mathrm{eV}$.

As we have emphasized, the modification of gravity is suppressed by $\Sigma$ or by the $\alpha\left(\vec{\nabla}^{2} \pi\right)^{2}$ term. However, we will argue below that with the nonlinear evolution, these also become important at the time scale $T_{\mathrm{NL}}$ found above because this is the timescale for the formation of caustic singularities. This is to be compared with the time scale for the modification of gravity in the linear regime, which is the time scale for the Jeans instability. If we take $M \lesssim 10 \mathrm{MeV}$ (as in Ref. [5]), the Jeans time scale is longer than the age of the universe, and the gravitational time scale of any object is a much shorter time scale. We see that the nonlinear effects completely dominate the dynamics in all regimes of interest.

\subsection{Fluid Picture}

We now show that the nonlinear equations of motion have a simple interpretation in terms of a perfect fluid if we neglect the $\alpha\left(\vec{\nabla}^{2} \pi\right)^{2}$ term in the action. As already mentioned in subsection 2.5, we can see this connection directly in terms of the stressenergy tensor for the ghostone mode. Near $X=1$ we can approximate $P(X)$ by

$$
P(X)=\frac{1}{8}(X-1)^{2}=\frac{1}{2} \Sigma^{2} .
$$

Neglecting the $\alpha\left(\vec{\nabla}^{2} \pi\right)^{2}$ term, the stress-energy tensor has the form

$$
T_{\mu \nu}=M^{4}\left\{-\frac{1}{2} \Sigma^{2} g_{\mu \nu}+\Sigma u_{\mu} u_{\nu}\right\},
$$


where

$$
u_{\mu}=\partial_{\mu} \phi
$$

Note that $u_{\mu}$ is nonzero and timelike everywhere. This has the form of the stressenergy tensor for a perfect fluid with 4 -velocity $u_{\mu}$. Because $u_{\mu}$ is the gradient of a scalar, the flow of the fluid is irrotational. The conservation of the stess-energy tensor $\nabla^{\mu} T_{\mu \nu}=0$ gives the equation of motion for the ghostone field, and also gives the Euler equation for the fluid. For $\Sigma \ll 1$ we can read off the density and pressure

$$
\rho=M^{4} \Sigma, \quad p=\frac{1}{2} M^{4} \Sigma^{2}=\frac{\rho^{2}}{2 M^{4}}
$$

This establishes the equivalence of the ghostone theory without the $\alpha\left(\vec{\nabla}^{2} \pi\right)^{2}$ term to the irrotational flow of a perfect fluid ${ }^{2}$.

It is also insightful to understand the equivalence directly in terms of the equations of motion. For the ghostone field, the equations of motion have the form of a conservation law

$$
\dot{\Sigma}=\vec{\nabla} \cdot[\Sigma \vec{\nabla} \pi]
$$

where $\Sigma$ is the charge density. For a fluid made of particles carrying the conserved charge, the current is $\vec{J}=\Sigma \vec{v}$, so we identify

$$
\vec{v}=-\vec{\nabla} \pi
$$

Again we see that the fluid flow is irrotational. In this fluid picture, the equations of motion are satisfied simply due to the fact that the fluid particles carry their charges with them. It remains only to satisfy the relation between $\Sigma$ and $\pi$ :

$$
\Sigma=\dot{\pi}-\frac{1}{2}(\vec{\nabla} \pi)^{2}-\Phi
$$

Taking the gradient of both sides and using Eq. (3.18) gives

$$
\frac{D \vec{v}}{D t}=-\vec{\nabla}(\Phi+\Sigma)
$$

\footnotetext{
${ }^{2}$ The Jeans length $\tilde{L}_{J}=2 \pi c_{s} / \sqrt{4 \pi G_{N} \rho}$ corresponding to this equation of state is $\tilde{L}_{J} \sim M_{P l} / M^{2}$, where $c_{s}=\sqrt{d p / d \rho}=\sqrt{\rho / M^{4}}$ is the sound velocity. Intriguingly, this agrees with the Jeans length (2.25) in the linear theory up to a constant of order unity. This equation of state ignores the $k^{4}$ term, while the linear analysis does not take into account the nonlinear term $\Sigma^{2}$ in $p$. Hence, it is not a priori clear whether these two Jeans length should be the same or not. Nonetheless, they agree.
} 
where

$$
\frac{D}{D t}=\frac{\partial}{\partial t}+\vec{v} \cdot \vec{\nabla}
$$

is the time derivative along the particle worldline (also called the convective or Lagrangian derivative). Eq. (3.19) is just Newton's law for a particle moving in a potential $\Phi+\Sigma$. Using the identifications Eq. (3.15) we can write

$$
-\vec{\nabla} \Sigma=-\frac{1}{\rho} \vec{\nabla} p
$$

which shows that Eq. (3.19) is precisely Euler's equation for the fluid. ${ }^{3}$

The equivalence between the ghostone dynamics and the dynamics of a perfect fluid was derived here in the nonrelativistic approximation and for linearized gravity. In fact, as shown in the appendix, it holds in full nonlinear general relativity.

We can now understand better the justification for neglecting the $\alpha\left(\vec{\nabla}^{2} \pi\right)^{2}$ term, which was used to derive the fluid picture. As discussed in the introduction, outside a gravitational source, the fluid (and therefore the ghostone field) responds on a time scale of order the gravitational infall time of the source. For reasonable values of $M$, this is much shorter than the time for the $\alpha\left(\vec{\nabla}^{2} \pi\right)^{2}$ term to become important. However, the particle trajectories in a perfect fluid tend to cross, leading to caustic singularities. (For $\Sigma \neq 0$, the pressure may stop these singularities, but they certainly occur for $\Sigma=0$.) At the caustic singularity, derivatives of the velocity field blow up, and higher-derivative terms such as the $\alpha\left(\vec{\nabla}^{2} \pi\right)^{2}$ term cannot be neglected. Therefore, the fluid picture is valid away from small regions where caustics form.

We can also understand better the extent to which the locally preferred frame (the 'aether') is dragged by the gravitational field. We expect the universe to consist of domains where $\Sigma$ is negligbly small and $\phi$ varies smoothy, separated by caustic regions. Away from the caustics, the locally preferred frame is a freely-falling frame, and in this sense the aether is dragged by the local gravitational field.

\subsection{Negative Energy}

We now re-examine the question of negative energy in the theory in the presence of the nonlinearities discussed above. Because the time translation invariance and $\phi$ shift invariance are spontaneously broken down to a diagonal time shift symmetry, there are different possible definitions of energy in this theory. Gravity couples to the

\footnotetext{
${ }^{3}$ Note that the equivalence between the ghostone and fluid pictures is a kind of duality, since it exchanges a constraint equation with an equation of motion.
} 
Noether charge of the original time translation symmetry, which we refer to as the 'gravitational energy.' This is not the same as the Noether charge associated with the time translation symmetry that is unbroken in the vacuum, which we call the 'inertial energy.'

The inertial energy $\varepsilon$ is the energy associated with the unbroken time translation symmetry of the Lagrangian Eq. (3.6). (It is also the Hamiltonian density of the system.) Conservation of inertial energy states that

$$
\dot{\varepsilon}=-\vec{\nabla} \cdot \vec{p}
$$

where

$$
\varepsilon=M^{4}\left\{\frac{1}{2} \Sigma^{2}+\frac{1}{2} \Sigma(\vec{\nabla} \pi)^{2}+\frac{\alpha}{2 M^{2}}\left(\vec{\nabla}^{2} \pi\right)^{2}\right\}
$$

is the inertial energy density and

$$
\vec{p}=M^{4}\left\{-\Sigma \dot{\pi} \vec{\nabla} \pi+\frac{\alpha}{M^{2}}\left[\left(\vec{\nabla}^{2} \pi\right) \vec{\nabla} \pi-\dot{\pi} \vec{\nabla}\left(\vec{\nabla}^{2} \pi\right)\right]\right\}
$$

is the momentum density. In the linearized approximation,

$$
\varepsilon=M^{4}\left\{\frac{1}{2} \dot{\pi}^{2}+\frac{\alpha}{2 M^{2}}\left(\vec{\nabla}^{2} \pi\right)^{2}\right\} \geq 0 .
$$

However, in the nonlinear theory the inertial energy is not positive definite due to the second term in Eq. (3.23). The energy density can be negative only in regions where $\Sigma<0$. In fact it is easy to see that the energy is unbounded from below. For example, for $\pi=c|\vec{x}|$ we have $\varepsilon=-\frac{1}{8} M^{4} c^{4}$.

We might expect this theory to be unstable because any region can lower its energy by emitting positive energy radiation to infinity. (Because inertial energy is positive in the linear theory, radiation must have positive energy.) The reason this does not happen is that there is another conserved quantity in the theory, namely the Noether charge associated with the $\phi$ shift symmetry. ${ }^{4}$ Because $\pi$ shifts under the shift symmetry, the conservation of shift charge is identical to the equation of motion for $\pi$ :

$$
\dot{\Sigma}=-\vec{\nabla} \cdot \vec{J}
$$

where $\Sigma$ is the shift charge density, and the shift current is

$$
\vec{J}=-\Sigma \vec{\nabla} \pi+\frac{\alpha}{M^{2}} \vec{\nabla}\left(\vec{\nabla}^{2} \pi\right) .
$$

\footnotetext{
${ }^{4}$ The gravitational energy is a linear combination of the inertial energy and the shift charge, so the conservation of the stress-energy tensor does not give an independent conservation law.
} 
If we neglect the $\alpha\left(\vec{\nabla}^{2} \pi\right)^{2}$ term, this conservation law is taken into account very directly in the fluid picture, to which we now turn. In this approximation, negative energy regions correspond precisely to regions where $\Sigma<0$, so we consider such a region surrounded by $\Sigma>0 .{ }^{5}$ In the fluid picture, the conserved charge is carried by the individual particles, so the $\Sigma<0$ region consists of particles with negative charge, while the boundary of the $\Sigma<0$ region consists of particles of vanishing charge. Therefore, there can be no flux of charge across the $\Sigma=0$ boundary, and the total charge inside the region does not change.

On the other hand, the $\Sigma=0$ boundary can move. Since $\vec{\nabla} \Sigma$ points outward at the boundary, the equation of motion for fluid particles Eq. (3.19) implies that the particles on the boundary experience an inward force due to the pressure. Therefore, the $\Sigma<0$ region tends to shrink.

These arguments show that the total shift charge integrated over a $\Sigma<0$ region

$$
Q=\int_{\Sigma<0} d^{3} x \Sigma
$$

does not change with time. This can also be seen from the fact that the shift current $\vec{J}$ vanishes on the $\Sigma=0$ boundary. The shift charge is not the same as the total inertial energy

$$
\mathcal{E}=\int_{\Sigma<0} d^{3} x \varepsilon
$$

However, if we neglect the $\alpha\left(\vec{\nabla}^{2} \pi\right)^{2}$ term, the flux of inertial energy across the $\Sigma=0$ boundary also vanishes, and therefore $\mathcal{E}$ also does not change with time.

As with the fluid picture, these results hold beyond the approximations made here. This is discussed in the appendix.

\subsection{Caustic Solutions}

The fluid picture can be used to understand the structure of the caustic singularties that occur when we neglect the $\alpha\left(\vec{\nabla}^{2} \pi\right)^{2}$ term. We restrict attention to the case $\Sigma=0$, where there is no pressure and the fluid particles follow geodesics. In this case, it is clear that there are caustics without the $\alpha\left(\vec{\nabla}^{2} \pi\right)^{2}$ term. It is possible that the pressure resolves the caustics in important situations such as inside galaxy halos made of ghostone dark matter, but we will not consider that here.

\footnotetext{
${ }^{5}$ We do not consider configurations that have $\Sigma<0$ at infinity. These can be eliminated by physical initial conditions. For example, in the expanding universe Hubble friction drives can drive $\Sigma \rightarrow 0$ from above.
} 
To introduce the subject of caustics, we consider the gravitational potential due to a uniform sphere of matter with density $\rho_{0}$. Inside the sphere, the gravitational potential is

$$
\Phi=\frac{\rho_{0}}{6 M_{\mathrm{Pl}}^{2}} r^{2}
$$

i.e. a harmonic potential. If we consider an initial fluid configuration where all particles are at rest, then all fluid particles reach the center of the sphere at the same time! This kind of 'perfect caustic' requires very special initial conditions and symmetry. Nonetheless, we will see that an analog of the perfect caustic appears to be responsible for the singularities that remain in the full theory even after the $\alpha\left(\vec{\nabla}^{2} \pi\right)^{2}$ term is included.

A more realistic situation will have less symmetry in the gravitational potential and the initial conditions. Nonetheless, in the presence of gravitational sources the fluid particle trajectories tend to cross, resulting in caustic singularities. The generic situation is that families of trajectories cross on a dimension- 2 surface. We want to zoom in on the behavior near the caustic surface, so we can treat the problem as 1-dimensional, and approximate the caustic surface by a plane. We also expect the potential to be irrelevant on the small scales where the caustic structure is important. This reduces the problem to a very simple 1-dimensional one.

In the absence of a potential, the particle trajectories are just straight lines:

$$
x=x_{0}+v_{0}\left(x_{0}\right) t
$$

Here we are labeling each trajectory by its initial position $x_{0}$ at $t=0$. The initial velocity function $v_{0}\left(x_{0}\right)$ determines the geodesics. The problem now is to find the velocity of the particle at a given point $(x, t)$, which is a simple kinematic problem.

To get a caustic near $x=0$, we are interested in the case where the particle trajectories that start near $x_{0}=0$ are focussed toward each other:

$$
v_{0}(0)=0, \quad v_{0}^{\prime}(0)<0 .
$$

For small $x_{0}$ we can therefore approximate $v_{0}\left(x_{0}\right)$ by a linear function of $x_{0}$ :

$$
v_{0}\left(x_{0}\right)=-\frac{x_{0}}{T}
$$

where $T$ is a constant that tells us how the initial velocity varies away from $x_{0}=0$. Solving Eq. (3.31) for $x_{0}$ we obtain

$$
x_{0}=-T \frac{x}{t-T}
$$




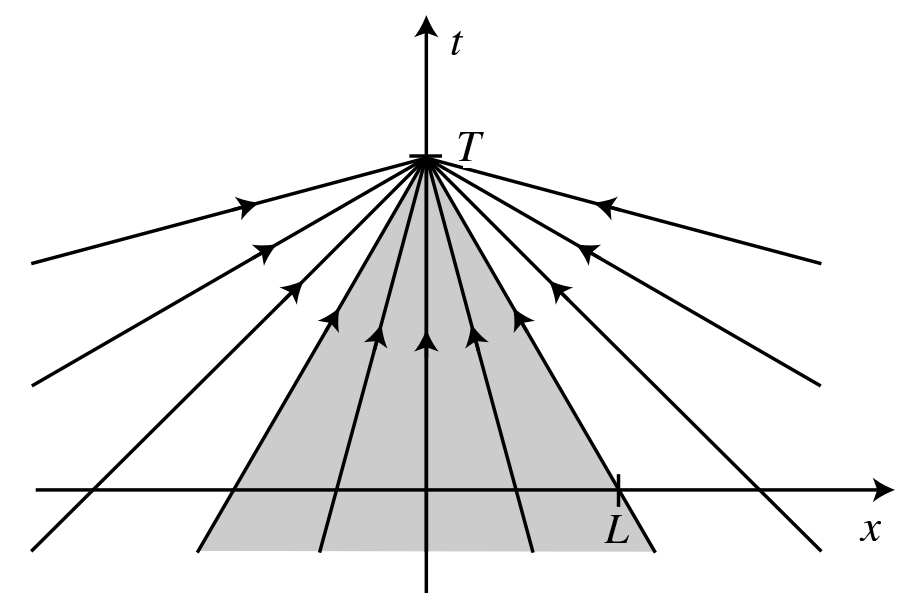

Fig. 4. The 1-dimensional 'perfect caustic.' The shaded regions shows the region where we can expand the solution perturbatively about the perfect caustic.

Since the velocity is constant along any trajectory, we have

$$
v(x)=v_{0}\left(x_{0}\right)=-\frac{x}{T-t} .
$$

We can relate this to the field $\pi$ using $\partial_{x} \pi=-v$, to obtain

$$
\pi(x, t)=-\frac{x^{2}}{2(t-T)}
$$

Note that this solution is scale-free, since $T$ just gives the time to the caustic. Shifting $t \rightarrow t-T$, we obtain

$$
\pi(x, t)=-\frac{x^{2}}{2 t}
$$

To understand this solution, note that the general condition for a caustic is

$$
\frac{\partial x}{\partial x_{0}}=0
$$

for fixed $t$. This gives the time to the caustic for a given value of $x_{0}$ as

$$
t_{\mathrm{c}}\left(x_{0}\right)=-\frac{1}{v_{0}^{\prime}\left(x_{0}\right)}
$$

For $v_{0}$ given by Eq. (3.33) above, we see that the caustic forms at $t=T$ for all initial points $x_{0}$. We call this the 'perfect caustic,' and it is illustrated in Fig. 4. 
In a realistic case, the function $v_{0}\left(x_{0}\right)$ will be more complicated. We want to expand about the point where the caustic first forms, i.e. the minimum of $t_{\mathrm{c}}\left(x_{0}\right)$, which we take to be $x_{0}=0$. Expanded about this point, $v_{0}$ takes the form

$$
v_{0}\left(x_{0}\right)=-\frac{x_{0}}{T}+\frac{1}{6 L^{2} T} x_{0}^{3}+\mathcal{O}\left(L^{-3}\right),
$$

where we have performed a boost so that $v_{0}(0)=0$. Because of the focussing of the geodesics, this expansion is valid only for

$$
|x| \ll L\left|\frac{t-T}{T}\right| .
$$

This region of validity is shown in Fig. 4.

We can now follow the same steps as for the perfect caustic, keeping higher order terms in Eq. (3.40). We find

$$
x_{0}=-T \frac{x}{t-T}-\frac{T^{3}}{6 L^{2}} \frac{x^{3} t}{(t-T)^{4}}+\mathcal{O}\left(L^{-3}\right) .
$$

and therefore

$$
v(x)=\frac{x}{t-T}+\frac{T^{3}}{6 L^{2}} \frac{x^{3}}{(t-T)^{4}}+\mathcal{O}\left(L^{-3}\right) .
$$

As above, this is related to the ghostone field solution by $\partial_{x} \pi=-v$.

This result can be used to evaluate the effect of the $\alpha\left(\vec{\nabla}^{2} \pi\right)^{2}$ term in the action near the caustic singularity. In the equation of motion for $\pi$, it contributes a term proportional to $\alpha \partial_{x}^{4} \pi$. This can be thought of as a restoring force, since it tends to smooth out the large gradients near the caustic region. Evaluating this in the caustic solution Eq. (3.43) gives

$$
\partial_{x}^{4} \pi=-\partial_{x}^{3} v=-\frac{T^{3}}{L^{2}} \frac{1}{(t-T)^{4}}+\mathcal{O}\left(L^{-3}\right) .
$$

Note that a nonzero contribution to $\partial_{x}^{4} \pi$ arises only at $\mathcal{O}\left(L^{-2}\right)$. In particular, there is no restoring force at all in the perfect caustic solution. We believe that this explains the singular behavior we find in numerical simulations below. Since the $\partial_{x}^{4} \pi$ vanishes in the perfect caustic, it is an exact solution to the full nonlinear equations, including the $\alpha\left(\vec{\nabla}^{2} \pi\right)^{2}$ term. This solution therefore becomes singular and exits the regime of validity of the effective theory. Even though the perfect caustic is a very special solution, it does describe the generic caustic close to the singularity. Also, the fact that the perfect caustic is a scale-free solution may mean that it is an attractor in the full nonlinear dynamics. 
We can use Eq. (3.44) to estimate the time and distance scale where the $\alpha\left(\vec{\nabla}^{2} \pi\right)^{2}$ term becomes important. This will happen when

$$
\alpha \frac{\partial_{x}^{4} \pi}{M^{2}} \gtrsim \ddot{\pi}
$$

For a solution that varies on a time scale $T$ and distance scale $L$, the scaling in subsection 3.2 shows that $\ddot{\pi} \sim L^{2} / T^{3}$. We therefore find that the $\alpha\left(\vec{\nabla}^{2} \pi\right)^{2}$ term becomes important for

$$
\Delta t \lesssim\left(\frac{\alpha T^{3}}{M L^{2}}\right)^{1 / 2}
$$

where $\Delta t=T-t$ is the time to the caustic. The distance scale where the $\alpha\left(\vec{\nabla}^{2} \pi\right)^{2}$ term becomes important is therefore

$$
\Delta x \sim \frac{L}{T} \Delta t \lesssim\left(\frac{\alpha T}{M}\right)^{1 / 2}
$$

Note that $\Delta x, \Delta t \gg M^{-1}$ as long as $L, T \gg M^{-1}$ and $L / T \ll 1$ (i.e. the system is nonrelativistic), so this is within the regime of validity of the effective theory.

\section{Numerical Simulations}

In this section we describe various numerical simulations of the ghost field which allow us to understand the rather exotic features of its non-linear evolution.

The test cases we first present assume symmetry to reduce the dynamics to a one dimensional problem, and will see that the various symmetries have different behaviours. We will discuss how caustics do indeed form in the theory. As mentioned earlier, this is clear for $\alpha=0$, but one would naively expect non-zero $\alpha$ to ameliorate this problem. However, as we will show, this is not the case, and for certain symmetries the 'perfect' caustic remains an attractor.

As one might expect, the singular behaviour becomes less strong as one moves from planar through axisymmetry to spherical symmetry. Indeed without any gravitational potential we will see the planar reduction exhibits singularities, while the spherical theory without potential does not. However, once a gravitationally attractive potential is added, all three symmetries become singular under evolution of regular initial data.

Clearly assuming symmetry can lead to unphysical behaviours, whilst the physical situation we are ultimately interested, namely structure formation, is expected 
to have very little symmetry. Hence this section will conclude with a study of a 3-d numerical evolution where no continuous symmetry is present and a moving gravitational potential seeds the ghost field growth. As expected from the 1-d examples, we again find singular caustics do develop, and interestingly appear to take a planar form.

\subsection{One dimensional evolutions: Planar, axial and spherical symmetry}

We will initially consider the case of evolution of the ghost field in the absence of gravitational sources, seeded instead from a local perturbation in the ghost field itself. Then reducing to planar symmetry $\pi=\pi(t, r)$ and we may write the equation for the ghost decoupled from gravity in a manifestly flux conservative form,

$$
\begin{aligned}
\dot{H} & =\partial_{r}\left[\Sigma+\frac{1}{2} H^{2}\right] \\
\dot{\Sigma} & =\partial_{r}\left[\Sigma H-L^{2} H^{\prime \prime}\right],
\end{aligned}
$$

where $H=\partial_{r} \pi$ is the gradient of the ghost field, $L=\sqrt{\alpha} / M$, the second line is simply the definition of $\Sigma$ and $\dot{x}, x^{\prime}$ are the time and space derivatives of $x$. We then use a Crank-Nicholson method to evolve our initial data, which we take to be a Gaussian profile in $\pi$

$$
\pi(t=0)=\pi_{0} e^{-r^{2}}
$$

with $\Sigma=0$ initially. As discussed previously, with $L=0 \Sigma$ would remain zero, but the higher derivative term sources $\Sigma$.

In order to make contact with the epoch of structure formation we wish to have moderate initial amplitudes so $\left|\pi_{0}\right|<1$ but is still of order unity. From (4.2) we have chosen units to have initial data with unit spatial variation. We then wish to have $L$, the ghost length scale, to be $L \ll 1$. Naturally numerical methods limit our ability to separate the initial data length scale from the ghost length whilst maintaining accuracy. However we may separate the scale sufficiently to see the asymptotic properties of taking $L$ very small.

The first figures we show, 5 and 6 , illustrate the generic behaviour for the planar system for small $L$, showing both $H$ and $\Sigma$ for the evolution. We show both the evolution of data for $L=0.005$ and also for $L=0$. for comparison, and the initial amplitude was taken to be $\pi_{0}=0.1$.

As expected $L=0$. evolution leads to a caustic, whose time for formation scales as $t_{\text {caustic }} \simeq 1 / \pi_{0}$. Adding the higher derivative term changes the evolution dramatically around this time scale, completely smoothing out the ghost field. However, we see 
from the figure that the radiated waves scattered by the action of this higher derivative term in fact are attracted back to the origin where they grow and become singular. Obviously since the symmetry is planar, this growth is not due to a measure factor, but rather is due a 'perfect caustic' forming, which as mentioned before cannot be rescued by the higher derivative term we use here. Indeed this perfect caustic formation can be seen locally in detail from $\Sigma$ near the singularity.

We note that taking sufficiently large $L \sim \sqrt{\pi_{0}}$ the field is completely scattered and the behaviour is essentially that of the linear theory and the non-linear terms never contribute to the evolution. However this large ghost length is clearly not compatible with our expectations for structure formation, where we expect $L \ll\left|\pi_{0}\right|<1$. For such parameter values the behaviour shown in these figures 5 and 6 is generic, and in figure 7 we plot the (inverse of) the time to the perfect caustic formation compared to $\pi_{0}$ for various $L$. As expected, this time increases with decreasing amplitude (eventually crossing over to the smooth linear evolution regime at $L \sim \sqrt{\pi_{0}}$ when $T \rightarrow \infty$ ), or with decreasing $L$ (so that the radiation from the increasingly perfect central caustic region is slower).

Thus for small $L$ a cartoon of the evolution is that the initially collapsing ghost field causes the higher derivative term to radiate strongly, but the process of radiation simply refines the collapsing region into the perfect caustic form where it eventually collapses.

Suitable modification of the conservative equations of motion (4.1) introduce the geometric measure factor associated with axisymmetry. In this case we find the behaviour essentially analogous to the planar case. Whilst the singular behaviour appears 'weaker', taking longer to reach the singularity for the same parameters, the singularity does indeed form. However moving to spherical symmetry we find a change in behaviour.

For spherical symmetry we again take the initial data (4.2) and evolve this in the absence of any gravitational potential. In figure 8 we plot the evolution of $H$ for $\pi_{0}=0.1$ and $L=0.005$ (as shown earlier for the planar case). The behaviour is clearly different with a totally non-singular evolution for all times, a portion of which is shown in the figure. Whilst for $L=0$. obviously the spherically symmetric ghost field exhibits the usual caustic singularity, we see the higher derivative term, aided by the geometric measure factor, can now radiate sufficient energy to avoid any later energy build up.

So far we have discussed the case of evolution of a local perturbation in the ghost field. We have seen that the symmetry of the initial data has a strong effect on whether the evolution is singular or not. Physically, however, we are interested more 



Fig. 5. Figure on the left shows $H=\partial_{r} \pi$ for planar evolution of initial Gaussian in $\pi$ with $\pi_{0}=0.1$ and $L=0$.. Note a caustic forms as expected. The figure on the right shows the same evolution but with non-zero $L=0.005$. Both evolutions are shown up to the time where the field becomes singular. We see the higher derivative term smooths the initial singularity present in the $L=0$. case but again results in a later singularity.

in the growth of the ghost field in response to a gravitational seed. Thus we must understand whether singularities in the ghost field form under these circumstances.

For planar symmetry we may add a gravitational potential to the system 4.1 by simply introducing a potential $\Phi$ to modify $\Sigma$ as,

$$
\dot{H}=\partial_{r}\left[\Sigma+\frac{1}{2} H^{2}+\Phi\right]
$$

We then use initial data for the evolution where both $H$ and $\Sigma$ are zero, and allow the gravitational potential to seed the growth in the ghost field. We find that for the weak potentials expected in our physical context, in the planar case this does not influence the behaviour appreciably. The gravitational source simply serves to produce a local non-zero configuration of the ghost field, which, once formed, then undergoes the singular evolutions described earlier, and is then largely independent of the presence of the potential. The same is true for axisymmetry.

The interesting case is clearly spherical symmetry, as without a potential we find 


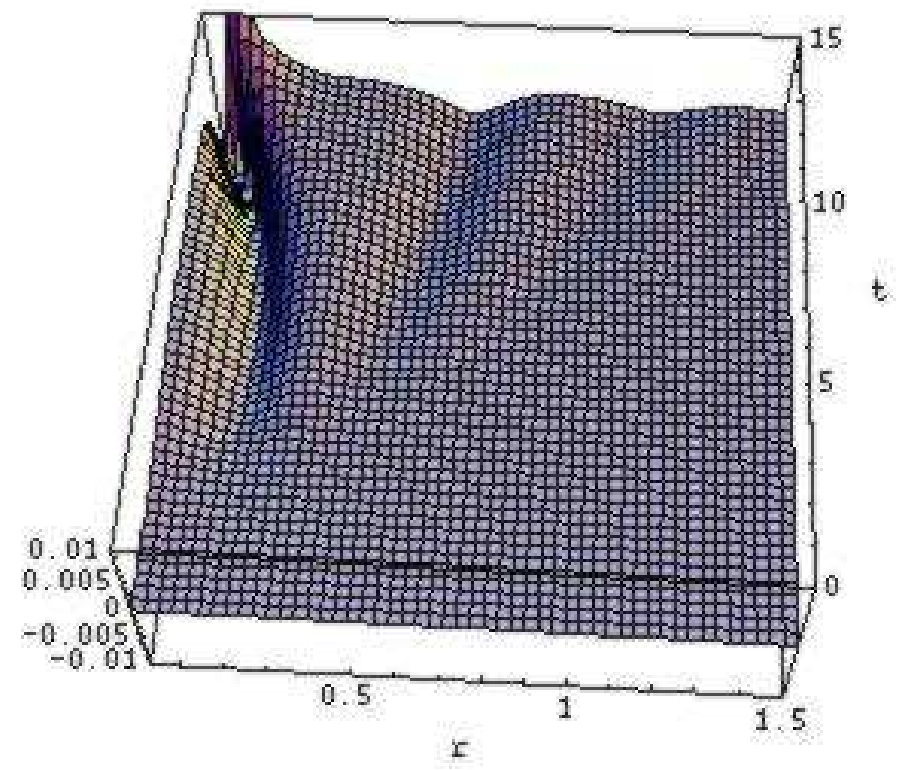

Fig. 6. Figure showing $\Sigma$ for the same evolution with $L=0.005$ as in figure 5 .

a non-singular evolution for smooth localized initial data. We take a potential such that,

$$
\nabla^{2} \Phi=\Phi_{0} e^{-r}
$$

In analogy with our earlier simulations $\Phi_{0}$ now acts like $\pi_{0}$ and we have taken units so the physical size of the source is of order unity. Then in figure 9 we show a typical evolution of $H$ and $\Sigma$ for parameters $\Phi_{0}=0.2$ with $L=0.02$.

We now see a complicated evolution where waves are radiated early on as the higher derivative term becomes important near the symmetry axis, but later these waves, consisting of positive and negative $\Sigma$ regions, are drawn grudgingly into the centre, eventually forming a singularity. This contrasts with the case without potential where such waves form, but are quickly radiated to infinity.

Thus the less singular behaviour present in spherical symmetry appears to be overcome once a gravitational potential is introduced and we conclude that, with the choice of higher derivative term in (4.1), the evolution of the ghost field in response to gravitational sources for all 3 reduced symmetries is singular. 


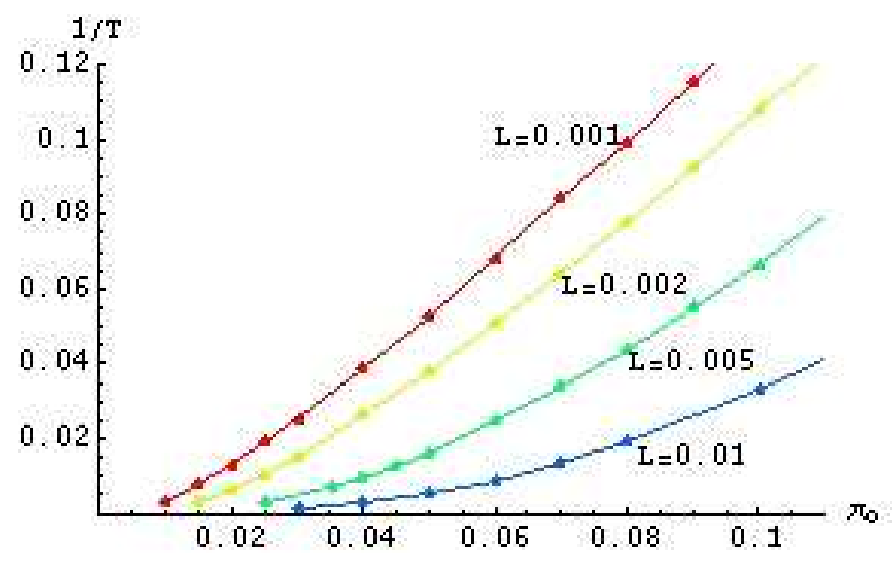

Fig. 7. Figure showing the inverse of the time to the 'perfect caustic' singularity, $1 / T$, against initial amplitude for various $L$ in planar symmetry.

\subsection{Three dimensional evolution}

We now test our claim that evolution is singular for gravitational seeds. In particular it is not obvious which symmetry will dominate the singularity evolution. For a point seed one might assume spherical symmetry to be the most relevant. However we have seen it is in the planar case that the singularity formation is quickest.

We evolve the $\pi$ field itself in three dimensional Cartesian coordinates using a simple explicit second order method. Clearly here resolution becomes an important issue and we are less able to separate the 2 length scales in the problem. However even with very modest resources and resolutions $\left(\simeq 150^{3}\right)$ we can achieve useful results.

Again we use initial conditions where $\pi$ and $\Sigma$ are zero. We seed the dynamics of the ghost field by taking a Gaussian potential,

$$
\Phi=-\Phi_{0} e^{-r^{2}}
$$

where now $r^{2}=\left(x-x_{0}(t)\right)^{2}+\left(y-y_{0}(t)\right)^{2}+z^{2}$ and,

$$
x_{0}(t)=0.7 \cos t \quad y_{0}(t)=0.7 \sin t
$$

so our gravitational seed executes a circular motion in the $z=0$ plane. Hence our system now has no continuous symmetries although there is a preferred plane, and also the geometry of the source is locally spherical. Therefore we may gain information about the local geometry of any singularities that form.

Figures 10, 11 and 12, show $\pi$ in the $x=0$ plane at various times for a typical evolution with parameters $\Phi_{0}=2$. and $L=0.2$ (note the physical scales are not 


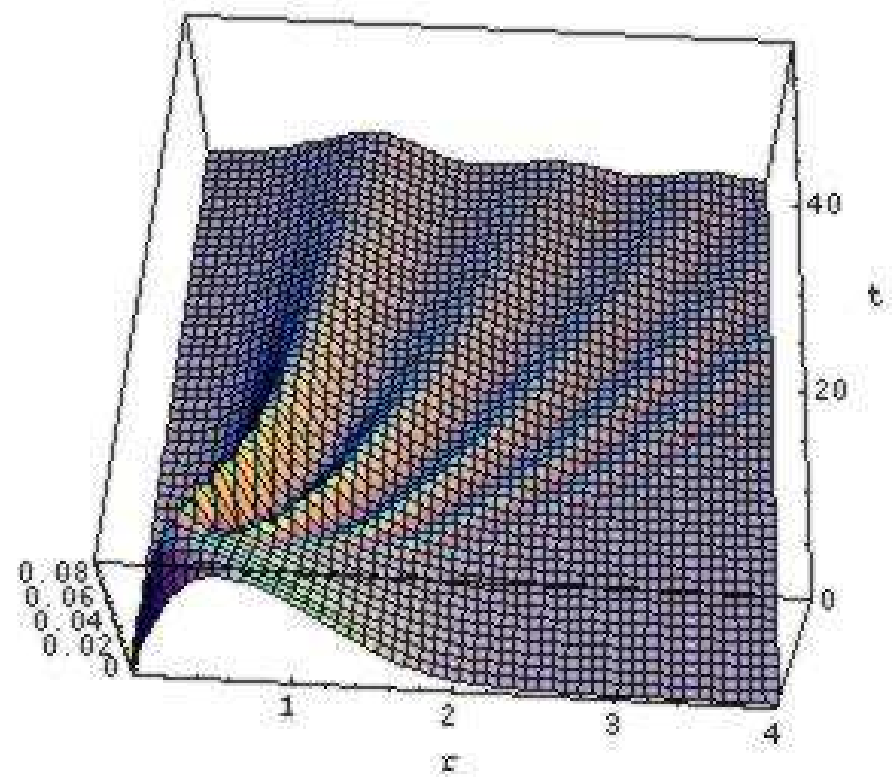

Fig. 8. Plot of $H=\partial_{r} \pi$ for spherical symmetry with no gravitational potential. Initial data is as in the previous planar case, and again the simulation shown has $\pi_{0}=0.1$ and $L=0.005$. We see no sign of singularity formation here, and indeed continued time evolution confirms this.

so well separated as in the one dimensional examples). The $y=0$ slices look very similar. We see that, as indicated from our study of the reduced dimension examples above, a singularity does indeed form, the last time frame being immediately before the configuration becomes singular. For the same static source, so $x_{0}(t)=y_{0}(t)=0$, the singularity occurs at $t \simeq 3.1$, but for the moving seed described here, it occurs at $t \simeq 6.2$, and the source executes approximately one revolution.

We see from the figures, and also the complete 3 -d data, that the singularity develops in the $z=0$ plane in which the source moves, and appears to have a planar form, filling the disc bounding the motion of the source. This is despite the fact that the source only completes one 'orbit' and thus is sufficiently slow moving that this disc symmetry is not obviously imposed. Thus we take this as evidence confirming our naive expectation that the local geometry of the singularity will be the symmetry where singularity formation is strongest, namely planar. 

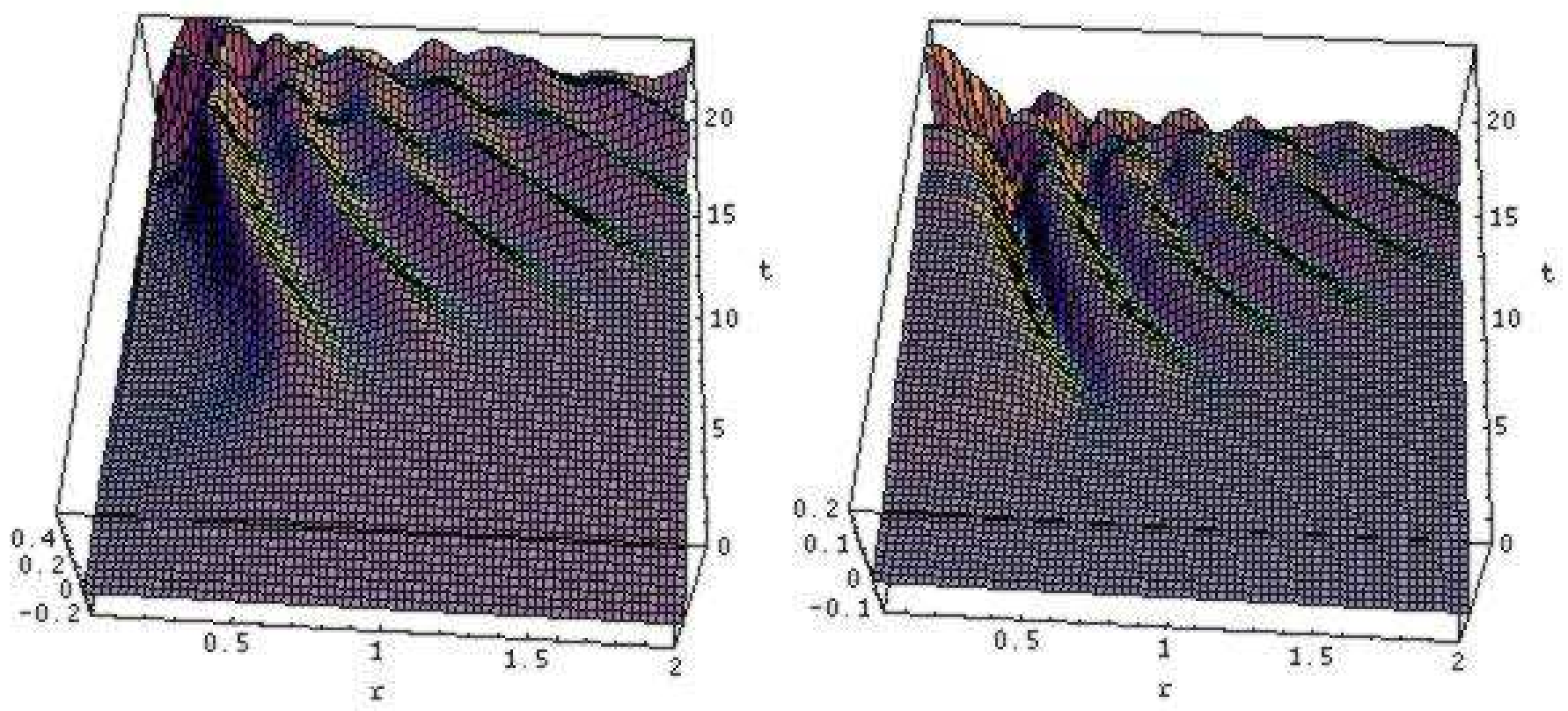

Fig. 9. Plot of $H=\partial_{r} \pi$ and $\Sigma$ for spherical symmetry with a gravitational potential. We see that a singularity does eventually form, whereas earlier we saw that with no potential, smooth localized initial data evolves in a non-singular manner for spherical symmetry.

\subsection{Interpretation of numerics}

In subsection 3.4 we have shown that a negative $\Sigma$ region does not grow but shrinks asymptotically. To be precise, the acceleration of the boundary of a negative energy region is towards the negative region itself. It has also been argued in subsection 3.5 that the perfect caustic is an attractor. With numerical results at hand, it is easy to confirm them. In this subsection, for simplicity we illustrate the confirmation in the planar case only, while the same qualitative features are seen also in the spherical case with the potential.

It is certainly worth while asking how caustics will form during structure formation. Here, we define a caustic in the broad sense as a region where $\Sigma$ becomes $O(1)$. Our understanding of caustics is still primitive and this question is obviously beyond the scope of this paper, but we can at least say that a caustic region does not swallow the whole space. The essential reason for this is the existence of a conserved charge $\int \Sigma d v$ resulting from the shift symmetry: if $|\Sigma|$ grows then the volume shrinks. In particular, we have shown and numerically confirmed that a negative $\Sigma$ region shrinks. In this sense caustics in the ghost condensate are somewhat similar to shockwaves 



Fig. 10. Figure showing $\pi$ in the $x=0$ plane at time $t=2.0$ for a full 3 -d evolution. The ghost field is sourced by a slowly moving gravitational potential, orbiting in the $z=0$ plane. The intersection points of the orbit with the slice shown are indicated by red dots in the contour plot. The peak in the field reflects the recent passage of the source through the plane.

in hydrodynamics. For a shockwave in hydrodynamics, we do not need to specify microscopic physics, such as atomic physics, to describe its dynamics and influence to macroscopic physics outside. Therefore, it is expected that we should be able to predict what happens outside the caustic region without too many assumptions about a UV completion in the caustics. In the next subsection we shall discuss possible UV completions.

Based on our numerical result, we expect that a planar caustic, being pretty much like a pancake or a disk, forms during structure formation. Indeed, the 3-d simulation showed that the initial stage looks spherical but deviations from spherical symmetry grow and the system evolves towards a planar shape.

As future work, we should ask at what scale caustics forms first in realistic situations. In particular, it is interesting to ask whether (i) a large caustic forms first and cascades to smaller ones via fragmentation or (ii) many small caustics form and buildup to large caustics. In either case, we might be tempted to speculate that the distribution of caustics should somehow trace the matter distribution since the for- 

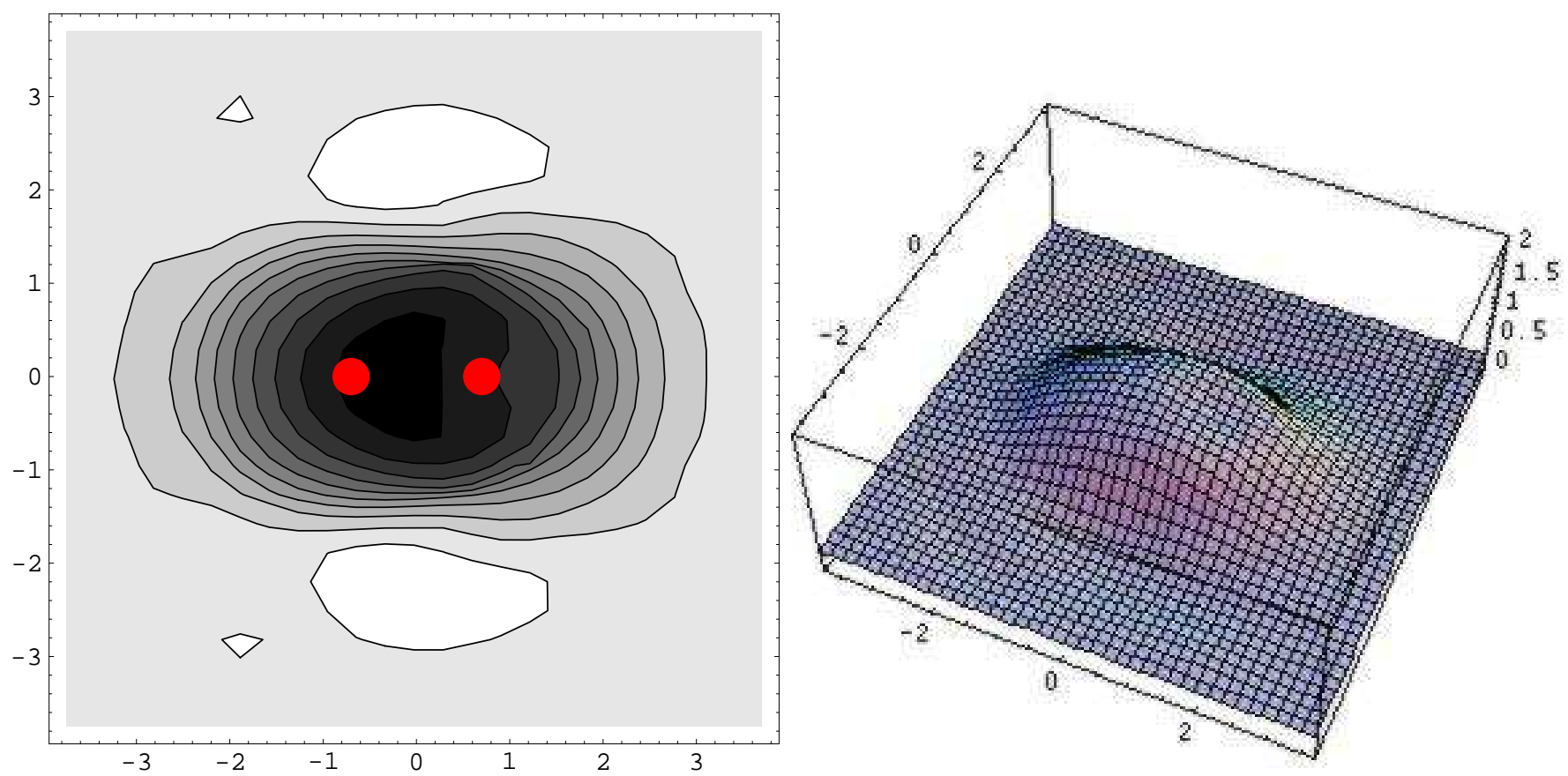

Fig. 11. Figure showing an intermediate time slice of $\pi$ at $t=4.0$ for the same $3-\mathrm{d}$ evolution as in the previous figure.

mation of caustics is enhanced by the gravitational potential due to matter. Clearly, we need more detailed analysis to see if this is the case or not. For this purpose we of course need to specify the initial condition of $\pi$ and the matter distribution, which determines the external gravitational potential. To be specific, let us suppose ghost inflation [8] happened at $H \simeq 10^{-4} M$. This is sufficient to explain the amplitude of primordial density perturbations $\delta \rho / \rho \simeq 10^{-5}$, which determines the initial conditions for matter, or equivalently the gravitational potential. We also have primordial perturbations of $\pi$. Thus, the problem to be solved is well-defined at least in principle. The nonlinear dynamics of the matter- $\pi$ coupled system with this initial condition certainly deserves further investigation.

\subsection{Possible UV completion/extension: gauged ghost and/or new terms}

We have seen that the nonlinear dynamics leads to formation of caustics. For the perfect caustics, the $\nabla^{4} \pi$ term in the equation of motion vanishes and, thus, does not act as a leading spatial derivative term. This is the very reason why the perfect caustic does not bounce. At the same time, this means that the spatial derivative expansion in this background starts differently from that in the trivial $\pi=0$ background and, 



Fig. 12. Continuing from the previous figures this plot shows the time slice $t=6.1$, just before the field becomes singular. Note the geometry of the almost singular field appears planar, and is roughly confined in the disc bounded by the source motion.

thus, new terms should be included in the leading Lagrangian for the perfect caustic background. It is also possible to consider new degrees of freedom which become relevant as the system approaches the caustic singularity.

With this in mind, for practical purposes (such as numerical studies of the ghost condensate as a dark matter candidate) it is probably useful to have simple models of new terms and/or new degrees of freedom which manifestly cutoff the singular behavior. Implementations adopted in this subsection are not particularly based on a consistent derivative expansion nor a more fundamental theory. Nonetheless, models here may be useful for practical purposes and, at least, show the existence of regular systems without caustics.

First, we consider the introduction of new degrees of freedom by turning on the gauge coupling for the gauged condensation proposed in ref. [15]. (Inclusion of new terms will be considered later in this section.) The gauged ghost condensation has new degrees of freedom, but they decouple from $\pi$ in the limit of zero gauge coupling. Hence, by promoting the gauge coupling constant to a function of $\pi$ 's derivatives it is possible to make the new degree relevant only near the singularity. 
The leading Lagrangian for the gauged ghost condensation is obtained by gauging the shift symmetry and is of the following form.

$$
\mathcal{L}=\frac{M^{2}}{2}\left(\mathcal{A}_{0}-M \Phi-\frac{1}{2 M} \overrightarrow{\mathcal{A}}^{2}\right)^{2}-\frac{1}{4 g^{2}} F_{\mu \nu} F^{\mu \nu}-\frac{\alpha}{2}(\vec{\nabla} \cdot \overrightarrow{\mathcal{A}})^{2} .
$$

In the $g^{2} \rightarrow 0$ limit, $F_{\mu \nu}$ goes to zero and, thus, $\mathcal{A}_{\mu}$ is written as a derivative of a scalar: $\mathcal{A}_{\mu}=\partial_{\mu} \pi$. Therefore, in this limit the original (ungauged) ghost condensation is recovered. In ref. [15] the nonlinear dynamics of the gauged ghost condensation was analyzed. It was shown that gauging the ghost condensation resolves the caustics for the spherically symmetric case with an external gravitational force. On the other hand, the caustics in the perfectly planar symmetric case remains unresolved. It was argued that small fluctuations on top of the perfectly planar symmetric layer should grow and lead to fragmentation of the layer into small pieces and caustics with codimension more than one should not form after that. Thus, it was argued that the gauged ghost condensation does not lead to caustics except for extremely fine-tuned situations. In the following we shall implement the gauged ghost condensation as a possible UV completion. As a very simple implementation, the gauge coupling is turned on when and only when $\overrightarrow{\mathcal{A}^{2}}$ is large. We shall see that this indeed cuts off the caustics in the spherically symmetric case with external gravitational force. On the other hand, we do not expect to cutoff the caustics in the perfectly planar symmetric case since the gauged ghost condensation itself does not bounce the perfectly planar symmetric caustics. However, as argued in ref. [15] and stated above, a planar symmetric caustics with perturbation is expected to fragment into small pieces and to bounce eventually.

With the spherical symmetry, the equation of motion is reduced to

$$
\begin{aligned}
& \dot{\Sigma}=\frac{1}{r^{2}}\left\{r^{2}\left(\Sigma \xi-b\left[\frac{1}{r^{2}}\left(r^{2} \xi\right)^{\prime}\right]^{\prime}\right)\right\}^{\prime}, \\
& \dot{\xi}=G^{2} b f+\Sigma^{\prime}+\xi \xi^{\prime}+\Phi^{\prime} \\
& \dot{f}=-\frac{1}{b}\left\{\Sigma \xi-b\left[\frac{1}{r^{2}}\left(r^{2} \xi\right)^{\prime}\right]^{\prime}\right\}
\end{aligned}
$$

where

$$
\begin{gathered}
\Sigma \equiv \frac{1}{2}(X-1), \quad \xi \equiv D_{r} \phi, \quad f \equiv \frac{1}{G^{2} b} \frac{F_{t x}}{M L_{p h y s}}, \\
b=\frac{\alpha}{\left(M L_{\text {phys }}\right)^{2}}, \quad G=g M L_{\text {phys }} .
\end{gathered}
$$

We can eliminate $\Sigma$ from the set of equations essentially because the electric field $f$ 
is determined solely by the charge. Actually, since

$$
\left[\frac{b}{r^{s}}\left(r^{s} f\right)^{\prime}+\Sigma\right]=0,
$$

the combination in the squared bracket is independent of $t$. Since the expansion of the universe in the early universe dilutes $\Sigma$ and $f$ to zero with an extremely high precision, we set

$$
\left.\Sigma\right|_{t=0}=\left.f\right|_{t=0}=0
$$

Hence, we obtain

$$
\Sigma=-\frac{b}{r^{2}}\left(r^{2} f\right)^{\prime}
$$

and $\Sigma$ can be eliminated from the set of equations. The result is

$$
\begin{aligned}
& \dot{\xi}=G^{2} b f-b\left[\frac{1}{r^{2}}\left(r^{2} f\right)^{\prime}\right]^{\prime}+\xi \xi^{\prime}+\Phi^{\prime}, \\
& \dot{f}=\frac{1}{r^{2}}\left(r^{2} f\right)^{\prime} \xi+\left[\frac{1}{r^{2}}\left(r^{2} \xi\right)^{\prime}\right]^{\prime} .
\end{aligned}
$$

We have described the gauged ghost condensation proposed in ref. [15]. Now we promote $G^{2}$ from a constant to a function of $\xi^{2}$ so that the gauge coupling is turned on when and only when the gradient becomes sufficiently large. For simplicity we choose

$$
G^{2}=\frac{G_{0}^{2}}{2}\left[1+\tanh \left(\frac{\xi^{2}-\xi_{0}^{2}}{\delta^{2}}\right)\right],
$$

where $G_{0}, \xi_{0}$ and $\delta$ are constants. Numerical simulation with $\alpha=2.0 \times 10^{-4}, G_{0}^{2}=$ $1.0 \times 10^{3}, \xi_{0}=1.0 \times 10^{-1}$ and $\delta=5.0 \times 10^{-4}$ is shown in Fig. 13. Fig. 14 compares this case with the singular result for the ungauged ghost condensation, i.e. $G_{0}=0$.

Now, let us turn our attention to possible new terms which manifestly cutoff the singular behavior. For this purpose it is useful to consider the nonlinear Hamiltonian of the system and to seek new terms which have positive contribution to the Hamiltonian. For simplicity we restrict our consideration to terms depending on $z \equiv(\nabla \pi)^{2}$ only.

To the leading Lagrangian $L_{0}=(X-1)^{2} / 2-\alpha\left(\nabla^{2} \pi\right)^{2}$, let us add a new term of the form $-F(z)$ :

$$
L=\frac{1}{2}(X-1)^{2}-\alpha\left(\nabla^{2} \pi\right)^{2}-F(z), \quad X-1=2 \dot{\pi}-z, \quad z=(\nabla \pi)^{2} .
$$

The Hamiltonian, or the generator of the unbroken diagonal part of (time translation symmetry $) \times($ the shift symmetry $)$, is

$$
H=2 \dot{\pi}^{2}-\frac{1}{2} z^{2}+\alpha\left(\nabla^{2} \pi\right)^{2}+F(z) .
$$



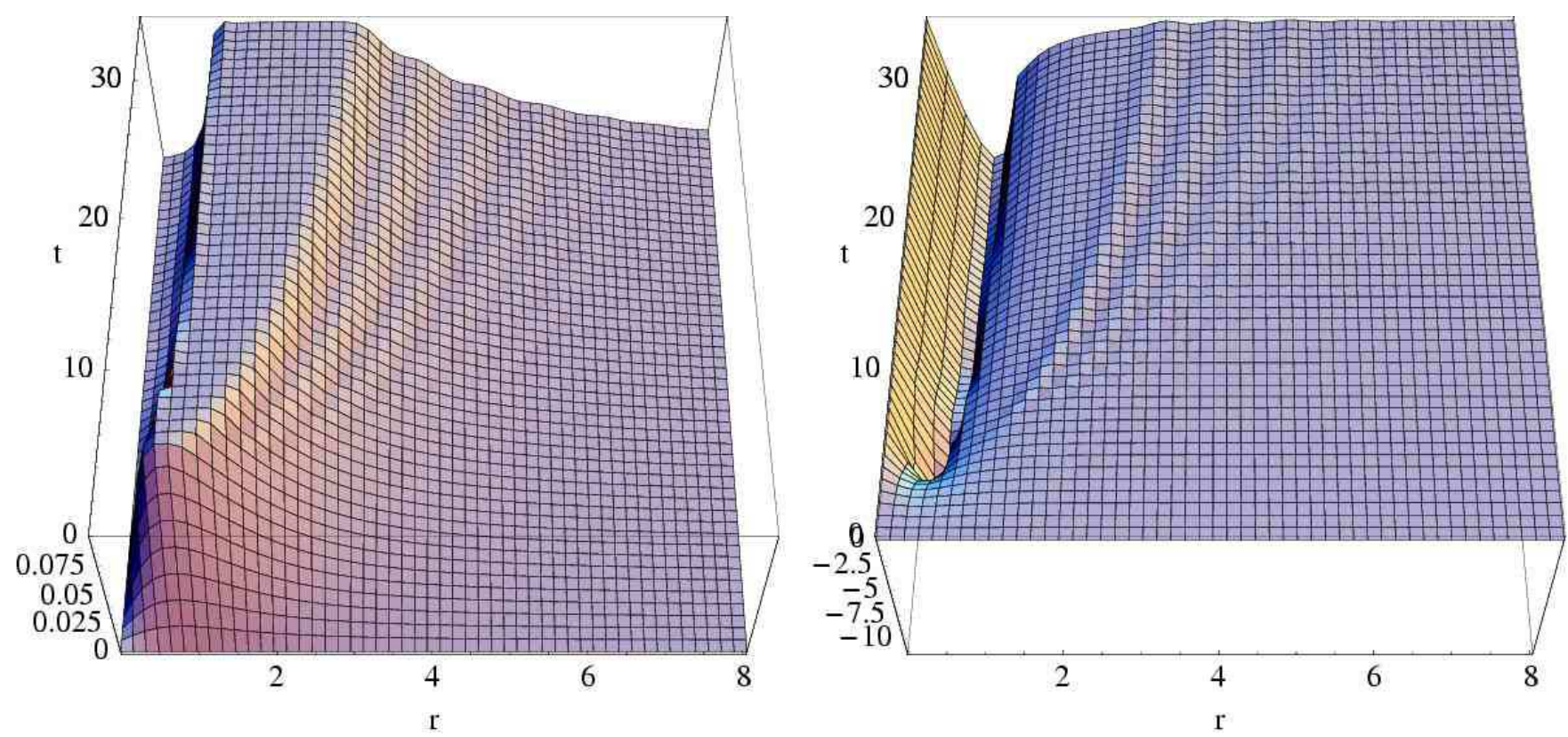

Fig. 13. The result of numerical simulation with $\alpha=2.0 \times 10^{-4}, G_{0}^{2}=1.0 \times 10^{3}$, $\xi_{0}=1.0 \times 10^{-1}$ and $\delta=5.0 \times 10^{-4}$. The left figure shows $\xi$. The right figure shows $f$. It is easy to see in the left figure that $|\xi|$ larger than $\xi_{0}$ is cut off by the gauge coupling. It is also easy to see in the right figure that the implosive accumulation of incoming waves is supported by the repulsive electric force $(f<0)$ near the center.

The second term $-z^{2} / 2$ has negative sign and, thus, the Hamiltonian would be unbounded from below without new terms. Actually, this is the origin of the nonlinear instability. Hence, in order to stabilize the system we would like to make the Hamiltonian bounded from below by choosing the new term appropriately. This is indeed the case if $F(z)$ increases faster than $z^{2} / 2$ for large $z$. On the other hand, $F(z)$ must not change physics below cutoff $M$ and, thus, must be turned off for small $z$. In particular, it must at least satisfy $F^{\prime}(0)=0$.

Just to show that this argument based on the Hamiltonian works, let us consider a simple choice

$$
\tilde{F}(z)=\frac{\gamma}{2} z^{2}\left[\tanh \left(\frac{z-\beta}{\delta}\right)+1\right],
$$

where $\beta, \gamma$ and $\delta$ are positive constants, and $\tilde{F}(z) \equiv 4 \sqrt{z}\left(\sqrt{z} F^{\prime}(z)\right)^{\prime}$. We have specified $\tilde{F}(z)$ instead of $F(z)$ just for practical purpose: since the equation of motion includes the term $\tilde{F}(z) \nabla \pi \cdot \nabla z / 2 z, \tilde{F}(z)$ is easier to be implemented in numerical codes than $F(z)$ itself. Results for the perfectly planar symmetric case with and without the new term are shown in Fig. 15. It is easy to see that the would-be caustics at 



Fig. 14. Comparison of the regular result for $G_{0}^{2}=1.0 \times 10^{3}$ with the singular result for $G_{0}^{2}=0$. The left figure shows a closer look at the regular behavior of $\xi$ shown in Fig. 13. The right figure shows the singular behavior of $\xi$ for $G_{0}^{2}=0$. The value of $\alpha$ and the external gravitational potential $\Phi$ are the same as before. Note that the scale of hight is different for these two figures.

$t \simeq 7.5$ is resolved by the new term.

In this subsection we considered simple models of new degrees of freedom and new terms arising near the perfect caustics. As stated in the beginning of this subsection, they must be taken into account since the usual leading spatial derivative term $\nabla^{4} \pi$ vanishes for the perfect caustics and the spatial derivative expansion in this background should start differently from that in the $\pi=0$ background. We have seen that turning on the gauge coupling of gauged ghost condensation or/and the new term resolves the singular behavior. We must, however, consider the simple models presented here just as an existence proof of systems without the singular behavior. A detailed analysis of the consistent derivative expansion and/or more realistic models of UV completion are necessary as a future work.

\section{Bounds}

In this section, we consider the bounds on the ghost condensate. 

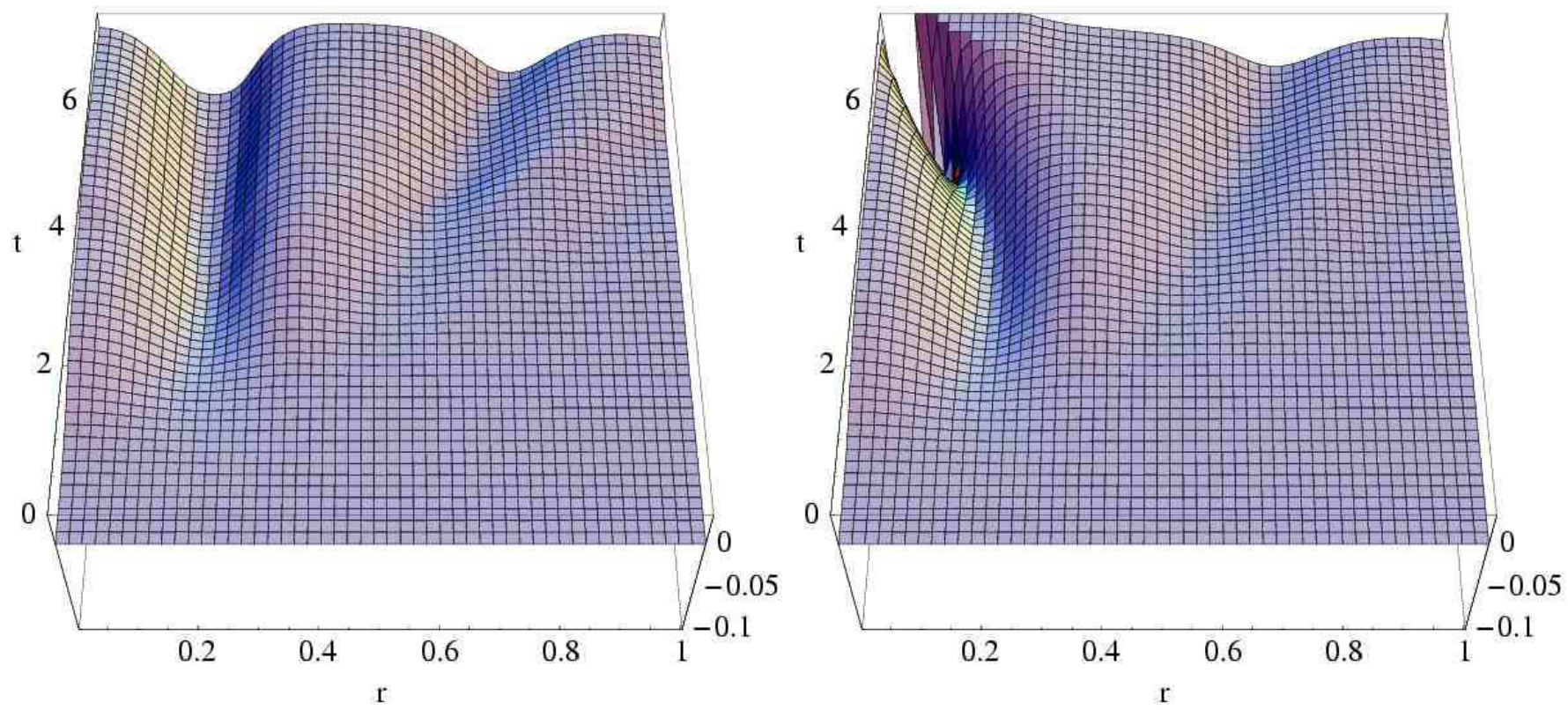

Fig. 15. The evolution of $X-1$ in the perfectly planar symmetric case with and without the new term. The left figure shows the evolution with the new term with $\beta=0.04, \gamma=150$ and $\delta=0.001$. The right figure shows the evolution without the new term. The ghost length scale $L_{\text {ghost }}$ is set to be 0.01 and the initial condition at $t=0$ is set by $\pi=-0.2 \times \exp \left(-x^{2}\right), X-1=0$. It is evident that the would-be caustics at $t \simeq 7.5$ is resolved by the new term.

\subsection{Jeans Instability}

For $M \gtrsim 10 \mathrm{MeV}$, the Jeans instability time is shorter than the lifetime of the universe, and we must consider the effects of this instability. We have seen that the nonlinear effects dominate near interesting gravitational sources, but the linear dynamics still controls the behavior of the system for sufficiently weak ghostone amplitudes. In the linear regime, fluctuations with wavelength $\lambda \gtrsim L_{\mathrm{J}}$ grow on a time scale

$$
\tau \sim T_{\mathrm{J}} \frac{\lambda}{L_{\mathrm{J}}}
$$

where

$$
L_{\mathrm{J}} \sim \frac{M_{\mathrm{Pl}}}{M^{2}}, \quad T_{\mathrm{J}} \sim \frac{M_{\mathrm{Pl}}^{2}}{M^{3}}
$$

are the Jeans length and time scales. Wavelengths of order $L_{\mathrm{J}}$ become unstable first, and longer wavelengths take longer to grow. Since fluctuations on wavelength shorter 
than $L_{\mathrm{J}}$ are stable, we expect the minimum size of a positive or negative energy region to be $L_{\mathrm{J}}$. On the other hand, the maximum size is determined by requiring that the time scale $\tau$ above be shorter than the Hubble time. Hence, a positive or negative region can grow within the age of the universe if its size $L$ is in the range

$$
L_{\mathrm{J}} \lesssim L \lesssim L_{\max }
$$

where

$$
L_{\max } \sim \frac{M}{M_{\mathrm{Pl}} H_{0}} \sim R_{\odot}\left(\frac{M}{100 \mathrm{GeV}}\right)
$$

The unstable modes grow at least until nonlinear effects become important. This happens for $\pi \gtrsim \pi_{\mathrm{c}}$, where

$$
\pi_{\mathrm{c}} \sim \frac{\lambda^{2}}{\tau}
$$

or equivalently $\Sigma \gtrsim \Sigma_{\mathrm{c}}$ with

$$
\Sigma_{\mathrm{c}} \sim \frac{\pi_{\mathrm{c}}}{\tau} \sim \frac{\lambda^{2}}{\tau^{2}} \sim \frac{M^{2}}{M_{\mathrm{Pl}}^{2}} .
$$

It is reasonable to assume that the nonlinear effects cut off the Jeans instability at this critical amplitude. This mechanism will fill the universe with regions of positive and negative ghostone field with amplitude of order $\pm \Sigma_{\mathrm{c}}$ and the size in the range Eq. (5.3). Since $\Sigma$ is a conserved charge, there will be equal amounts of positive and negative $\Sigma$.

The sun's Newtonian potential triggers the Jeans instability of the ghost condensate and, thus, it is expected that there be a positive or negative region around the sun. This is justified if the 'aether' is efficiently dragged by the sun and we now argue that this is indeed the case. To do this, it is useful to work in the rest frame of the sun. Far from the sun, the aether is moving with constant velocity $v \sim 10^{-3}$, but near the sun the velocity field will be distorted by the presence of the sun. By using the fluid picture of the ghostone field, we estimate the effect on a fluid particle with speed $v$ and impact parameter $r$. The fluid particle will be a distance of order $r$ away for a time $\Delta t \sim r / v$, so the change in the particle velocity in the impulse approximation is

$$
\Delta v \sim \frac{R_{\mathrm{S}}}{r^{2}} \cdot \frac{r}{v} \sim \frac{R_{\mathrm{S}}}{v r}
$$

where $R_{\mathrm{S}}$ is the Schwarzschild radius of the source. Thus, the change in the velocity of a fluid particle becomes comparable to or greater than the initial velocity if $r<r_{\mathrm{drag}}$, where

$$
r_{\mathrm{drag}} \sim \frac{R_{\mathrm{S}}}{v^{2}}
$$


For our sun, $r_{\text {drag }} \sim 10 R_{\odot}$, so the dragged region extends outside the solar radius. ${ }^{6}$

We require that the absolute value of the mass of the lump with the critical density $\rho_{c}$ and the size $L_{\max }$ be at worst less than the solar mass:

$$
\rho_{c} L_{\max }^{3} \lesssim M_{\odot}
$$

This requirement gives the bound

$$
M \lesssim 10^{3} \mathrm{GeV}
$$

Since the high power of $M$ (the l.h.s. $\propto M^{9}$ ) is involved in Eq. (5.9), a more stringent requirement on the mass of the lump will not substantially improve the bound.

\subsection{Twinkling from Lensing}

We have argued that if $M \gtrsim 10 \mathrm{MeV}$, then the Jeans instability fills the universe with regions of positive and negative energy of size $L \gtrsim L_{\mathrm{J}} \sim M_{\mathrm{Pl}} / M^{2}$ with energy density $\rho_{\mathrm{c}} \sim M^{6} / M_{\mathrm{Pl}}^{2}$. This will happen everywhere, in particular in the voids between galaxies. Any light that travels to us from far away will therefore be lensed by these positive and negative regions. These positive and negative energy regions move, because the local rest frame of the lensing regions is different from that of our galaxy, so the result is that the observed luminosity of any point source will change with time. This is similar to the twinkling of the stars in the night sky caused by time dependent temperature differences in the atmosphere. In this subsection, we work out the bounds on the ghost condensate from this effect.

Suppose that the universe is filled with regions of positive and negative energy with size $L$ and density $\rho_{\mathrm{c}}$. A light ray traveling through such a region will lens by an angle

$$
\Delta \theta \sim \Phi \sim \frac{\rho_{\mathrm{c}} L^{2}}{M_{\mathrm{Pl}}^{2}} \sim \frac{M^{6} L^{2}}{M_{\mathrm{Pl}}^{4}} .
$$

If a light ray travels a distance $d \gg L$, then it will undergo $N \sim d / L$ uncorrelated lensing events, so the total angular deviation will be enhanced by a $N^{1 / 2}$ random walk factor:

$$
\Delta \theta_{\mathrm{tot}} \sim\left(\frac{d}{L}\right)^{1 / 2} \frac{M^{6} L^{2}}{M_{\mathrm{Pl}}^{4}} .
$$

We see that the largest angular deviation comes from the largest $L$ and largest $d$.

\footnotetext{
${ }^{6}$ This radius is still much less than the orbital radius of Mercury.
} 
The size of $L$ is limited by the time for the Jeans instability to form as in (5.3). If the source is the cosmic microwave background, then $d \sim H_{0}^{-1}$ and we obtain

$$
\Delta \theta_{\mathrm{CMB}} \sim \frac{M^{15 / 2}}{M_{\mathrm{Pl}}^{11 / 2} H_{0}^{2}} \sim\left(\frac{M}{100 \mathrm{GeV}}\right)^{15 / 2}
$$

for the largest regions with the size $L \sim L_{\max }$. The high power of $M$ makes the precise experimental limit on $\Delta \theta_{\mathrm{CMB}}$ irrelevant, and we obtain the bound

$$
M \lesssim 100 \mathrm{GeV}
$$

For $M \sim 100 \mathrm{GeV}$, the size of the largest critical region is $L \sim 10^{12} \mathrm{~cm}$, approximately the radius of the sun. The local velocity of these regions relative to our galaxy is of order $10^{-3}$, so the time scale for one of these regions to cross the line of sight is of order a day, which is therefore the time scale of the variation.

If there is a distant astrophysical source that is observed to shine with very little time variation, it may give a competitive bound. But given the high power of $M$ involved, it seems difficult to improve on this bound significantly.

\subsection{Supernova time-delay}

Gravitational lensing considered in the previous subsection induces a time-delay for light-rays coming from far distances. With this time-delay effect, observed supernovae should be older than they appear. Thus, this effect would change the estimate of dark energy by observation of Type Ia supernovae. Since the determination of the dark energy by supernovae observation is known to be consistent with the WMAP data, we require that the time-delay is sufficiently shorter than the total time:

$$
\frac{\Delta t}{t} \sim(\Delta \theta)^{2} \sim \frac{M^{6} L_{\max }^{2}}{M_{\mathrm{Pl}}^{4}} \leq 1
$$

Note that the precise experimental limit on the $\Delta t / t$ is irrelevant because of the higher power of $M$ involved in the l.h.s. From this we obtain the bound

$$
M \lesssim 10^{3} \mathrm{GeV}
$$

\subsection{Nonlinear Energy Loss}

A gravitating object moving relative to the preferred frame will lose energy. As dis-

cussed above, the ghostone field outside a gravitating object is in the nonlinear regime 
for essentially all gravitationally interesting objects and interesting values of $M$. In this subsection, we estimate this energy loss in the nonlinear regime. Interestingly, we obtain parametrically the same result as the linear calculation, and so this is a small effect in the real world.

It is simplest to work in a frame where the ghost fluid is at rest. As the gravitating object moves through the medium, its gravitational potential excites the surrounding ghost fluid, and therefore the object loses energy. This picture is very similar to the energy loss of charged particles in a medium, treated in standard texts on electrodynamics. In fact, the particle picture makes the analogy very close.

Consider then a gravitating object characterized by its Schwarzschild radius $R_{\mathrm{S}}=$ $M_{*} / M_{\mathrm{Pl}}^{2}$ moving with speed $v$ in the $+z$ direction relative to the preferred frame. We estimate the effect on a particle with impact parameter $r$ using the impulse approximation in order to see the response of the ghost condensate in the particle picture. The moving object will be a distance or order $r$ away for a time $\Delta t \sim r / v$, so the change in the particle velocity in the impulse approximation is

$$
\Delta v \sim \frac{R_{\mathrm{S}}}{r^{2}} \cdot \frac{r}{v} \sim \frac{R_{\mathrm{S}}}{v r}
$$

In terms of the ghostone field, this means that we generate a disturbance with $\vec{\nabla} \pi \sim$ $\Delta v$. To estimate the energy density associated with this, we must remember that there is no modification of gravity if we neglect the $\alpha\left(\vec{\nabla}^{2} \pi\right)^{2}$ term. The change in the ghostone field energy is therefore

$$
\Delta \rho \sim \alpha M^{2}\left(\vec{\nabla}^{2} \pi\right)^{2} \sim \frac{\alpha M^{2} R_{\mathrm{S}}^{2}}{v^{2} r^{4}}
$$

The total energy transferred to the ghostone fluid is therefore

$$
\dot{E} \sim v \frac{d E}{d z} \sim v \int d r r \frac{\alpha M^{2} R_{\mathrm{S}}^{2}}{v^{2} r^{4}}
$$

This integral is dominated at small $r$, so we have

$$
\dot{E} \sim \frac{\alpha M^{2} R_{\mathrm{S}}^{2}}{v r_{\min }^{2}} .
$$

We now consider the short distance cutoff $r_{\text {min }}$. One cutoff comes from the use of the impulse approximation. This is valid only if the particles moves a distance smaller than $r$ in the time $\Delta t$ :

$$
a(\Delta t)^{2} \sim \frac{R_{\mathrm{S}}}{r^{2}}\left(\frac{r}{v}\right)^{2} \lesssim r
$$


which gives

$$
r_{\min } \sim \frac{R_{\mathrm{S}}}{v^{2}}
$$

For $r \sim r_{\min }$ we have $\Delta v \sim v$, so the maximum velocity of particles with $r<r_{\min }$ is $\Delta v \sim v$. This shows that the contribution of particles with $r<r_{\min }$ is suppressed, justifying the use of $r_{\min }$ as a cutoff.

Another cutoff is provided by the finite size $R$ of the object itself. This is smaller than $r_{\text {min }}$ given above provided that

$$
\frac{R_{\mathrm{S}}}{R} \gtrsim v^{2}
$$

The left-hand side is the surface gravity of the object, and the right-hand side is of order $10^{-6}$ for a typical astrophysical object. For example, the sun has a surface gravity of order $10^{-5}$, while the earth has a surface gravity of order $10^{-9}$.

Putting this together, we see that for an object of sufficiently strong surface gravity (Eq. (5.23)), the energy loss is

$$
\dot{E} \sim \alpha M^{2} v^{3}
$$

Note that the dependence on the mass of the object (as well as the Planck mass) has dropped out. Eq. (5.24) is the same parametric formula obtained from a linear calculation, and completely negligible for macroscopic objects. If the surface gravity is weak, the energy loss is even smaller.

\section{Ghost Dark Matter}

As shown in Ref. [5] and reviewed above, the expansion of the universe drives $\Sigma \rightarrow 0$. If $\Sigma$ is nonzero early in the history of the universe, then the ghostone energy density evolves with the scale factor $a$ as

$$
\rho \sim \Sigma \sim a^{-3}
$$

with $p \sim \rho^{2} / M^{4} \ll \rho$ for $\rho \ll M^{4}$. (Since the energy density of the universe at matter-radiation equality is $\sim(1 \mathrm{eV})^{4}$, this requres $M \gtrsim 1 \mathrm{eV}$.) Thus, the ghostone energy and pressure redshifts just like cold dark matter. Note that since $\Sigma$ is a conserved charge density, a nonzero value of $\Sigma$ early in the universe corresponds to a nonzero charge asymmetry, similar to the baryon asymmetry in the universe. In such a scenario, the amount of dark matter is determined by very different microphysics 
from the conventional freeze-out of particle dark matter. In particular, if we want to generate a nonzero density of $\Sigma$ charge from an initial condition with no charge asymmetry ('ghostogenesis'), the theory must contain interactions that violate the shift symmetry. We will not discuss the required initial conditions for ghost dark matter further, but instead turn to the viability of this scenario.

As already noted in Ref. [5], small fluctuations of the ghostone field with $\delta \rho / \rho \ll 1$ also behave just like cold dark matter. This can be seen from the fact that the equation of motion in both cases follows from the conservation of the stress-energy tensor for linearized scalar fluctuations, which is the same for the ghostone field as for cold dark matter. Using the results of the present paper, we can see explicitly that the equations of motion for the ghostone mode are equivalent to a perfect fluid with equation of state $p=\rho^{2} / 2 M^{4}$ even in the nonlinear regime $\delta \rho / \rho \sim 1$.

However, the ghostone fluid behaves very differently from cold dark matter in the nonlinear regime. To see this, let us recall the basic facts about the nonlinear dynamics of cold dark matter. An overdense region of cold dark matter starts gravitational collapse, and decouples from the universe expansion. The cold dark matter particles do not reach the center of the overdense region because they carry angular momentum, and there is no efficient mechanism for them to lose it. The trajectories of cold dark matter particles generically cross, but nothing special happens because the particles have negligible interactions. The over-dense region therefore virializes, becoming effectively a cloud of dark matter particles orbiting in the gravitational potential produced by all the other dark matter particles. At any given point in the overdense region, there are streams of dark matter particles orbiting in many different directions. Numerical simulations show that this kind of dark matter is quite successful in reproducing many of the observed features of structure in our universe, although there are open questions and possible discrepancies. (For a recent review, see e.g. Ref. [16].)

In the case of ghost dark matter, the trajectories of the 'particles' in the fluid picture cannot cross, since the theory is microscopically a scalar field. The pressure term will provide a repulsive force in the over-dense regions, and a simple argument suggests that it is more important than higher derivative terms such as the $\alpha\left(\vec{\nabla}^{2} \pi\right)^{2}$ term in the action. In a region where ghostone energy is the dominant gravitational source, the gravitational potential is given by

$$
\vec{\nabla}^{2} \Phi=m^{2} \Sigma
$$

where

$$
m=\frac{M^{2}}{\sqrt{2} M_{\mathrm{Pl}}} \sim \frac{1}{L_{\mathrm{J}}} .
$$


The equation of motion of the fluid particles is

$$
\frac{D \vec{v}}{D t}=-\vec{\nabla}(\Phi+\Sigma)
$$

where the $\vec{\nabla} \Sigma$ term gives rise to the pressure force. From this we see that the pressure force will dominate over the gravitational forces if $\Sigma \gtrsim \Phi$. In the case where the dominant source of gravity is $\Sigma$ (as in a halo of ghost dark matter), Eq. (6.2) shows that $\vec{\nabla} \Sigma \gtrsim \vec{\nabla} \Phi$ for lumps smaller than $m^{-1} \sim L_{\mathrm{J}}$. This means that the pressure force will act to smooth out any inhomogeneities on scales smaller than $L_{\mathrm{J}}$.

To see that the pressure force can indeed balance gravitational forces, we can consider spherically symmetric static configurations, i.e. 'ghost stars.' The force-free condition is $\vec{\nabla}(\Phi+\Sigma)=0$, which implies $\Sigma=-\Phi+$ constant. If the constant is nonzero, then $\Phi \sim r^{2}$ at large $r$, which can be discarded because it has growing energy density at infinity. We therefore have $\Sigma=-\Phi$, and Eq. (6.2) gives a solution

$$
\Sigma=-\Phi=\Sigma_{0} \frac{\sin (m r)}{m r}
$$

where $\Sigma_{0}=\Sigma(r=0)$. For this solution the $\pi$ 's gradient grows, but its contribution to the energy density is suppressed by $M^{2} / M_{P l}^{2}$ and thus negligible. Actually, the gradient of $\pi$ at time $t$ after the formation of the 'ghost star' is estimated as

$$
\vec{\nabla}^{2} \pi \sim m^{2} \dot{\pi} t \sim m^{2} \Sigma_{0} t \sim m^{2} \frac{\sqrt{\rho_{0}}}{M_{P l}} \frac{t}{t_{d y n}},
$$

where $t_{d y n} \sim \frac{1}{\sqrt{G_{N} \rho_{0}}}$ is the dynamical time for the 'ghost star' and $\rho_{0}=M^{4} \Sigma_{0}$ is the central density. Thus, the energy due to the gradient is estimated as

$$
\Delta \rho \sim \alpha M^{2}\left(\vec{\nabla}^{2} \pi\right)^{2} \sim \alpha \frac{M^{2}}{M_{P l}^{2}} \rho_{0}\left(\frac{t}{t_{d y n}}\right)^{2} .
$$

Hence, $\Delta \rho / \rho_{0}$ remains suppressed by the factor $M^{2} / M_{P l}^{2}$ and is negligible for $t \sim t_{d y n}$. This means that the 'ghost star' can be considered as a quasi-static configuration. This solution has alternating regions of positive and negative $\Sigma$, and therefore does not describe a normal star. However, the region $r<\pi / m$ has positive $\Sigma$ (if $\Sigma_{0}>0$ ) and describes a region where gravity and pressure forces balance. In fact, we can easily check that the solution in this region is stable. The Lagrangian for fluctuations about this solution is

$$
\mathcal{L}=M^{4}\left[\frac{1}{2} \dot{\pi}^{2}-\frac{1}{2} \Sigma_{0} \frac{\sin (m r)}{m r}(\vec{\nabla} \pi)^{2}\right] .
$$


The equation of motion for spherical perturbations is

$$
\ddot{\pi}=\frac{\Sigma_{0}}{m r^{2}} \partial_{r}\left[r \sin (m r) \partial_{r} \pi\right] .
$$

Separating variables as $\pi=\tilde{\pi}(r) e^{-i \omega t}$, multiplying the equation of motion by $r^{2} \tilde{\pi}$, integrating from $r=0$ to $r=\pi / m$ and using integration-by-parts, we obtain

$$
\omega^{2} \int_{0}^{\pi / m}[\tilde{\pi}(r)]^{2} r^{2} d r=\frac{\Sigma_{0}}{m} \int_{0}^{\pi / m}\left[\tilde{\pi}^{\prime}(r)\right]^{2} r \sin (m r) d r .
$$

This implies that $\omega^{2}>0$ unless $\tilde{\pi}^{\prime}=0$ everywhere in the interval of integration. Hence, the positive $\Sigma$ region near the origin is stable. Although this solution is not realistic, it gives an explicit example where pressure can cancel gravitational attraction.

We see that in regions where $\Sigma>0$ is the dominant source of gravity, the pressure force tends to smooth out inhomogeneities on scales smaller than $L_{\mathrm{J}}$. Let us ask whether the $\alpha\left(\vec{\nabla}^{2} \pi\right)^{2}$ term is important in these regions. In the solution without the $\alpha\left(\vec{\nabla}^{2} \pi\right)^{2}$ term, we have

$$
\Sigma \sim \frac{\pi}{T} \sim \frac{\pi^{2}}{L^{2}}
$$

From this, we can see that the $\alpha\left(\vec{\nabla}^{2} \pi\right)^{2}$ term is negligible for

$$
L \gtrsim \frac{1}{M \sqrt{\Sigma}}
$$

This is satisfied at $L \sim L_{\mathrm{J}}$ provided that

$$
\Sigma \gtrsim \frac{M^{2}}{M_{\mathrm{Pl}}^{2}}
$$

Note that this is the critical amplitude $\Sigma_{\mathrm{c}}$ discussed in subsection 5.1. If we assume that the ghostone energy density $\rho \sim M^{4} \Sigma$ accounts for all dark matter in the universe, then Eq. (6.13) is always satisfied in the overdense regions for $M \lesssim 10 \mathrm{MeV}$. In that case, it is very plausible that the pressure is sufficient to smooth out the would-be caustics that occur during structure formation in our universe.

We emphasize that we are not claiming that the pressure prevents caustic singularities from forming during structure formation. For example, a collapsing $\Sigma>0$ region may 'bounce' and leave behind a region of $\Sigma \sim 0$ (a void) or even $\Sigma<0$. In these regions, the singularities discussed in the previous section may become important. Also, a very over-dense region may lead to caustics in a less dense region nearby. 
However, even if the $\alpha\left(\vec{\nabla}^{2} \pi\right)^{2}$ term becomes important in some small regions of the halo, leading to negative energy regions, we argued in the previous section that the negative energy regions tend to shrink and carry negligible energy and they happen at very small scales. Therefore, we do not expect them to be important in the overall distribution of energy.

It would certainly be very interesting to perform numerical simulations of the nonlinear dynamics of self-gravitating ghostone halos to see whether ghost dark matter can explain the structure of the universe we observe today.

\section{Conclusions}

Infrared modifications of gravity have potential connections to the puzzles we are facing in cosmology such as dark energy and dark matter. Such theories can be viewed as gravity in the Higgs phase. The simplest possibility with a single extra degree of freedom which nonlinearly realizes the broken time diffeomorphism is described by ghost condensation - a scalar field with a time dependent vacuum expectation value, $\phi \propto t$. In a previous paper [5], we have shown that a healthy low-energy effective theory can be constructed following the symmetry requirement. It is stable for small fluctuations in the absence of gravity $\left(M_{\mathrm{Pl}} \rightarrow \infty\right)$. When gravity is turned on, there is an infrared instability at long wavelengths and large time scale similar to the usual Jeans instability for ordinary matter in the presence of gravity. This potentially provides the strongest constraint on the UV cutoff of the effective theory.

The leading nonlinear interactions in the effective theory of ghost condensation are also fixed by the symmetry. By power counting, they are irrelevant operators so they are unimportant for small fluctuations about the ground state. However, they can become important in the presence of large classical sources. In this paper, we show that for realistic astrophysical gravity sources, the time scale for the nonlinear terms to become large is shorter than the time scale of linear propagations. Therefore, the nonlinear effects are dominant near these astrophysical bodies and this paper is devoted to studying these effects.

We show that there is a simple picture for the nonlinear evolutions of the 'ghost field.' They are simply described by the geodesics in the gravitational field of the source. The geodesic picture necessarily breaks down when geodesics cross and caustics form. At these points the higher derivative terms become important. From the numerical simulations we see that the generic caustics can be resolved by the leading higher derivative terms in the linear lagrangian. However, there exist perfect caustics which cannot be stopped by the leading higher derivative terms. Their resolution can 
only be addressed by the physics above the UV cutoff, beyond the regime of validity of the low-energy effective theory. A potential worry is whether some catastrophe may be caused by the negative energy region formed due to the caustics. We show that both analytically and in numerical simulations that the negative energy region shrinks with the total amount of energy inside the region staying approximately constant, so it will not swallow the whole space. In addition, for small enough $M$ the total energy inside each of these region is extremely small by astrophysics standards so it is not expected to give large anomalous gravitational effects. As long as such regions can be resolved in a full theory, we do not expect disastrous effects or strong bounds coming from these caustics.

In fact, the nonlinear effects may even relax the bound on the UV cutoff of the theory. In the linear approximation, we obtained a bound of $M \lesssim 10 \mathrm{MeV}$ from requiring the time scale of the Jeans instability to be longer than the age of the universe. The nonlinear effects can cut off the growth of the Jeans instability and hence removes the above bound. The total energy in a lump can only grow to $M_{\mathrm{Pl}}$ which is minuscule before the nonlinear dynamics cut off the growth. Now the question is how the positive and negative energy regions change the standard picture of our universe. We found that light rays coming from far distances lens by those lumps. By requiring that the random walk of light rays due to the sequence of lensing events does not smear out the observed CMB anisotropies, we found a slightly weaker bound of $M \lesssim 100 \mathrm{GeV}$.

Nonlinear dynamics will also play an important role if we try to imagine that dark matter is made of ghost condensate. We have shown that a coherent deviation of ghost condensation from the ground state redshifts like matter, and in the linear regime the growth of the perturbations is identical to the usual cold dark matter. Whether it can give rise to the correct structure formation in the universe depends on the nonlinear evolution. This is an interesting but complicated question, which may require detailed simulations. It is left for future studies.

In this paper, we have begun the investigation of the nonlinear dynamics of ghost condensation as a Higgs phase of gravity, which exhibits a rich structure. While we have obtained a qualitative picture of some of the dynamics, a more complete understanding is clearly desirable and deserves further investigations, as required for making more concrete connections with various cosmological questions. 


\section{Acknowledgments}

We would like to thank Paolo Creminelli, Lev Kofman and Matias Zaldarriaga for useful discussions. S. M. thanks Aspen Center for Physics where part of the work was done. N. A-H. is supported a David and Lucille Packard Foundation Fellowship for Science and Engineering, and by the DOE under contract DE-FG02-91ER40654. H.-C. C. and T. W. are supported by NSF grant PHY-0244821, M. A. L. is supported by NSF grant PHY-009954.

\section{Appendix: Higgs Phase of Full General Relativity}

\section{A.1 Fluid Picture}

We now show that the fluid picture derived in the main text holds in full general relativity, i.e. allowing relativistic motion and strong gravitational fields. In order to remain within the realm of validity of the effective theory, we must restrict ourselves to small ghostone ampltiudes, i.e.

$$
\Sigma \ll 1
$$

Neglecting the $\alpha$ term in the action, the equations of motion of the ghostone field can be written

$$
\nabla_{\mu} J^{\mu}=0
$$

where

$$
J^{\mu}=\Sigma u^{\mu}
$$

and

$$
u^{\mu}=\nabla^{\mu} \phi
$$

We cannot interpret $u^{\mu}$ as a 4 -velocity because $u^{2}=X \neq 1$ in general. However, this effect is $\mathcal{O}\left(\Sigma^{2}\right)$, as can be seen by defining a normalized 4 -vector $\hat{u}_{\mu}$ by

$$
\hat{u}^{\mu}=\frac{u^{\mu}}{\sqrt{X}}=u^{\mu}\left[1+\mathcal{O}\left(\Sigma^{2}\right)\right] .
$$

We can therefore interpret

$$
u^{\mu}=(\dot{\phi},-\vec{\nabla} \phi)
$$


as the 4-velocity of the fluid, up to $\mathcal{O}\left(\Sigma^{2}\right)$ corrections.

It now remains only to satisfy the relation between $\Sigma$ and $\phi$. Differentiating the relation

$$
g^{\mu \nu} \nabla_{\mu} \phi \nabla_{\nu} \phi=X=1+2 \Sigma,
$$

we obtain

$$
\nabla_{\mu} \Sigma=\frac{1}{2} \nabla_{\mu}\left(\nabla^{\rho} \phi \nabla_{\rho} \phi\right)=\nabla^{\rho} \phi \nabla_{\mu} \nabla_{\rho} \phi=\nabla^{\rho} \phi \nabla_{\rho} \nabla_{\mu} \phi
$$

or

$$
u^{\rho} \nabla_{\rho} u_{\mu}=\nabla_{\mu} \Sigma
$$

This has the form of the geodesic equation of a particle subject to an additional gradient force with potential $\Sigma$. For the special case $\Sigma=0$, the fluid particles follow geodesics. The velocity field satisfies the curl-free condition

$$
\nabla_{[\mu} u_{\nu]}=0
$$

implying that the flow is irrotational.

Conversely, any family of particle trajectories satisfying Eq. (A.9) with initial conditions

$$
u^{\mu} \mid=\text { given, } \quad \nabla_{[\mu} u_{\nu]} \mid=0
$$

on some initial value hypersurface defines a solution of the ghostone equations of motion. To see this, note that the vanishing of $\nabla_{[\mu} u_{\nu]}$ is preserved by the equation of motion Eq. (A.9). This can be seen from the identity

$$
u^{\rho} \nabla_{\rho}\left(\nabla_{[\mu} u_{\nu]}\right)=\left(\nabla_{[\nu} u_{\rho]}\right) \nabla_{\mu} u^{\rho}-\left(\nabla_{[\mu} u_{\rho]}\right) \nabla_{\nu} u^{\rho}
$$

which is derived as follows:

$$
\begin{aligned}
u^{\rho} \nabla_{\rho} \nabla_{\mu} u_{\nu} & =u^{\rho}\left(\nabla_{\mu} \nabla_{\rho} u_{\nu}-R_{\rho \mu \nu}{ }^{\sigma}\right) \\
& =-\nabla_{\mu} u^{\rho} \nabla_{\rho} u_{\nu}+\nabla_{\mu}\left(u^{\rho} \nabla_{\rho} u_{\nu}\right)-R_{\rho \mu \nu \sigma} u^{\rho} u^{\sigma} \\
& =-\nabla_{\mu} u^{\rho} \nabla_{[\rho} u_{\nu]}-\nabla_{\mu} u^{\rho} \nabla_{\nu} u_{\rho}+\nabla_{\mu} \nabla_{\nu} \Sigma-R_{\rho \mu \nu \sigma} u^{\rho} u^{\sigma}
\end{aligned}
$$

Antisymmetrizing in $\mu$ and $\nu$ then gives Eq. (A.12). Therefore, the velocity field $u_{\mu}$ can be written as a gradient $u_{\mu}=\nabla_{\mu} \phi$. The field $\phi$ now obeys the equation of motion Eq. (A.2), with

$$
g^{\mu \nu} \nabla_{\mu} \phi \nabla_{\nu} \phi=X+\text { constant. }
$$


The integration constant can be seen to vanish by imposing appropriate boundary conditions at infinity.

This means that many of the results derived in the main text hold in full general relativity. In particular, in regions where the solution is given by $\Sigma=0$, the local preferred frame given by $\phi$ is a freely falling frame. In this sense, the aether is 'dragged' even by strong gravitational fields.

\section{A.2 Negative Energy Region Shrinks}

We now show that any $\Sigma<0$ region shrinks in full general relativity, neglecting the $\alpha\left(\vec{\nabla}^{2} \pi\right)^{2}$ term in the equation of motion.

Now let us consider a hypersurface $\Sigma=0$ defining the boundary between a negative energy region $(\Sigma<0)$ and the surrounding positive energy region $(\Sigma>0)$. On the boundary $\Sigma=0$, the equation of motion is reduced to

$$
\partial_{\mu} \Sigma u^{\mu}=0
$$

Now let us calculate the acceleration $a^{\mu}=u^{\rho} \nabla_{\rho} u^{\mu}$. By differentiating the definition of $\Sigma$, we obtain

$$
\partial_{\mu} \Sigma=u^{\rho} \nabla_{\mu} u_{\rho}=u^{\rho} \nabla_{\rho} u_{\mu}
$$

where we have used $\nabla_{[\mu} u_{\rho]}=0$, and thus

$$
a^{\mu}=\partial^{\mu} \Sigma
$$

With this expression it is easy to know the direction to which the boundary hypersurface evolves:

$$
a^{\mu} \nabla_{\mu} \Sigma=\partial^{\mu} \Sigma \partial_{\mu} \Sigma<0
$$

Namely, the boundary hypersurface evolves towards the region with negative $\Sigma$. In other words, the negative energy region shrinks.

\section{A.3 Relativistic Caustic}

We now show how the caustic solutions discussed in subsection 3.5 can be found in an elegant way in the relativistic formulation. We consider the case of $1+1$ dimensions, which is relevant for the generic caustic. We have seen that the spacetime surface $\phi(x)=0$ is perpendicular to the family of geodesics. We can therefore use Gaussian normal coordinates based on this surface. In this coordinate system, $\phi$ is the 'time' variable, and the metric in the $(x, t)$ subspace is

$$
d s^{2}=d \phi^{2}-A^{2}(\phi, \sigma) d \sigma^{2}
$$


where the spatial variable $\sigma$ parameterizes the position along the $\phi=$ constant surfaces. Without loss of generality, we can choose $A(0, \sigma)=$ constant, since this corresponds to choosing the 'space' coordinate on the surface $\phi=0$ to be proper distance. The geodesics perpendicular to the surfaces of constant $\phi$ are simply $\sigma=$ constant in these coordinates.

The condition that the metric Eq. (A.19) is flat is

$$
\partial_{\phi}^{2} A=0
$$

(For example, one can check that this guarantees the vanishing of the Riemann tensor.) Imposing $A(0, \sigma)=1$, the general solution is

$$
A(\phi, \sigma)=\left[1-\phi / \phi_{\mathrm{c}}(\sigma)\right]
$$

where $\phi_{\mathrm{c}}(\sigma)$ is a function that specifies the distribution of velocities on the surface $\phi=0$. Note that the coordinate system breaks down when $\phi=\phi_{\mathrm{c}}(\sigma)$; this is a sign of the caustic singularity. For a caustic singularity

In this coordinate system, it is trivial to compute invariants such as

$$
\begin{aligned}
\square \phi & =\frac{1}{\sqrt{-g}} \partial_{\mu}\left[\sqrt{-g} g^{\mu \nu} \partial_{\nu} \phi\right]=\frac{1}{A} \partial_{\phi} A \\
& =\frac{1}{\tau},
\end{aligned}
$$

where

$$
\tau=\phi-\phi_{\mathrm{c}}
$$

is the time to the caustic. Similarly,

$$
\begin{aligned}
\square^{2} \phi & =\frac{1}{\sqrt{-g}} \partial_{\mu}\left[\sqrt{-g} g^{\mu \nu} \partial_{\nu} \square \phi\right]=\frac{1}{A} \partial_{\phi}\left[A \partial_{\phi} \square \phi\right]-\frac{1}{A} \partial_{\sigma}\left[A^{-1} \partial_{\sigma} \square \phi\right] \\
& =-\frac{1}{\tau^{3}}-\frac{\phi_{\mathrm{c}}\left[\left(\phi_{\mathrm{c}}^{\prime}\right)^{2}+\phi_{\mathrm{c}} \phi_{\mathrm{c}}^{\prime \prime}\right]}{\tau^{4}}+\frac{3 \phi_{\mathrm{c}}^{2}\left(\phi_{\mathrm{c}}^{\prime}\right)^{2}}{\tau^{5}} .
\end{aligned}
$$

Expanding the function $\phi_{\mathrm{c}}(\sigma)$ for small $\sigma$, we obtain

$$
\square^{2} \phi \sim-\frac{1}{\tau^{3}}-\frac{\phi_{\mathrm{c}}^{2}(0) \phi_{\mathrm{c}}^{\prime \prime}(0)}{\tau^{4}}+\mathcal{O}\left(\sigma^{2}\right)
$$

This agrees precisely with the results of subsection 3.5 in the nonrelativistic limit, with the identifications $\sigma=x_{0}, \phi_{\mathrm{c}}(\sigma)=t_{\mathrm{c}}$, and $\tau=t-T$. 


\section{References}

[1] R. Gregory, V. A. Rubakov and S. M. Sibiryakov, "Opening up extra dimensions at ultra-large scales," Phys. Rev. Lett. 84, 5928 (2000) [arXiv:hep-th/0002072]; I. I. Kogan, S. Mouslopoulos, A. Papazoglou, G. G. Ross and J. Santiago, "A three three-brane universe: New phenomenology for the new millennium?," Nucl. Phys. B 584, 313 (2000) [arXiv:hep-ph/9912552]; I. I. Kogan and G. G. Ross, "Brane universe and multigravity: Modification of gravity at large and small distances," Phys. Lett. B 485, 255 (2000) [arXiv:hep-th/0003074]; I. I. Kogan, S. Mouslopoulos, A. Papazoglou and G. G. Ross, "Multi-brane worlds and modification of gravity at large scales," Nucl. Phys. B 595, 225 (2001) [arXiv:hepth/0006030]; K. Freese and M. Lewis, "Cardassian Expansion: a Model in which the Universe is Flat, Matter Dominated, and Accelerating," Phys. Lett. B 540, 1 (2002) [arXiv:astro-ph/0201229]; J. W. Moffat, "Modified gravitational theory as an alternative to dark energy and dark matter," arXiv:astro-ph/0403266.

[2] G. R. Dvali, G. Gabadadze and M. Porrati, "4D gravity on a brane in 5D Minkowski space," Phys. Lett. B 485, 208 (2000) [arXiv:hep-th/0005016]; G. R. Dvali and G. Gabadadze, "Gravity on a brane in infinite-volume extra space," Phys. Rev. D 63, 065007 (2001) [arXiv:hep-th/0008054]; C. Deffayet, G. R. Dvali and G. Gabadadze, "Accelerated universe from gravity leaking to extra dimensions," Phys. Rev. D 65, 044023 (2002) [arXiv:astro-ph/0105068]; G. Gabadadze and M. Shifman, "Softly massive gravity," Phys. Rev. D 69, 124032 (2004) [arXiv:hep-th/0312289].

[3] N. Arkani-Hamed, H. Georgi and M. D. Schwartz, "Effective field theory for massive gravitons and gravity in theory space," Annals Phys. 305, 96 (2003) [arXiv:hep-th/0210184]; M. A. Luty, M. Porrati and R. Rattazzi, "Strong interactions and stability in the DGP model," JHEP 0309, 029 (2003) [arXiv:hepth/0303116]; A. Nicolis and R. Rattazzi, JHEP 0406, 059 (2004) [arXiv:hepth/0404159].

[4] S. M. Carroll, V. Duvvuri, M. Trodden and M. S. Turner, "Is cosmic speed-up due to new gravitational physics?," arXiv:astro-ph/0306438.

[5] N. Arkani-Hamed, H.-C. Cheng, M. A. Luty and S. Mukohyama, "Ghost condensation and a consistent infrared modification of gravity," JHEP 0405, 074 (2004) [arXiv:hep-th/0312099]. 
[6] V. A. Rubakov, "Lorentz-violating graviton masses: Getting around ghosts, low strong coupling scale and VDVZ discontinuity," arXiv:hep-th/0407104; M. V. Libanov and V. A. Rubakov, "More about spontaneous Lorentz-violation and infrared modification of arXiv:hep-th/0505231; B. M. Gripaios, "Modified gravity via spontaneous symmetry breaking," JHEP 0410, 069 (2004) [arXiv:hep-th/0408127]; S. L. Dubovsky, "Phases of massive gravity," JHEP 0410, 076 (2004) [arXiv:hep-th/0409124]; S. L. Dubovsky, P. G. Tinyakov and I. I. Tkachev, "Massive graviton as a testable cold dark matter candidate," arXiv:hep-th/0411158; S. L. Dubovsky, P. G. Tinyakov and I. I. Tkachev, "Cosmological attractors in massive gravity," arXiv:hep-th/0504067.

[7] J. D. Bekenstein, "Modified gravity vs dark matter: Relativistic theory for MOND," arXiv:astro-ph/0412652.

[8] N. Arkani-Hamed, P. Creminelli, S. Mukohyama and M. Zaldarriaga, "Ghost inflation," JCAP 0404, 001 (2004) [arXiv:hep-th/0312100].

[9] S. Mukohyama, "Black holes in the ghost condensate," arXiv:hep-th/0502189.

[10] A. V. Frolov, "Accretion of ghost condensate by black holes", Phys. Rev. D 70, 061501 (2004) [arXiv:hep-th/0404216].

[11] G. N. Felder, L. Kofman and A. Starobinsky, "Caustics in tachyon matter and other Born-Infeld scalars," JHEP 0209, 026 (2002) [arXiv:hep-th/0208019].

[12] D. Krotov, C. Rebbi, V. A. Rubakov and V. Zakharov, "Holes in the ghost condensate," Phys. Rev. D 71, 045014 (2005) [arXiv:hep-ph/0407081].

[13] S. L. Dubovsky, "Star tracks in the ghost condensate," JCAP 0407, 009 (2004) [arXiv:hep-ph/0403308].

[14] M. Peloso and L. Sorbo, "Moving sources in a ghost condensate," Phys. Lett. B 593, 25 (2004) [arXiv:hep-th/0404005].

[15] H. C. Cheng, M. A. Luty, S. Mukohyama and J. Thaler, "Spontaneous Lorentz breaking at high energies," to appear in JHEP [arXiv:hep-th/0603010].

[16] J. P. Ostriker and P. J. Steinhardt, "New light on dark matter," Science 300, 1909 (2003) [arXiv:astro-ph/0306402]. 